JAN 163a - 0

\title{
Weakest Preconditions for Progress
}

\author{
Johan J. Lukkien, Jan L.A. van de Snepscheut \\ Computer Science \\ California Institute of Technology \\ Pasadena, CA 91125
}

26 March 1991

\begin{abstract}
Predicate transformers that map the postcondition and all intermediate conditions of a command to a precondition are introduced. They can be used to specify certain progress properties of sequential programs.
\end{abstract}

\section{Introduction}

The semantics of a program notation defines the meaning of each program written in that notation. The semantics describes relevant aspects of the execution of the programs by a computer. Execution of a program may be viewed as a, possible nonterminating, sequence of state transitions. Program semantics described by $w l p$ and $w p$ predicate transformers (cf. [3,4]) relates initial states and final states, if any. One of the attractive aspects of this style of semantic definitions is that it supports the development of programs: program verification and construction boil down to calculation with predicates, sometimes referred to as equational reasoning (cf. [3,5]).

The two predicate transformers $w l p$ and $w p$ relate initial and final states only and are independent of the intermediate states reached by program execution. The advantages are many, both to the programmer and to the implementer of the program notation. In some cases, however, properties of the intermediate states are of interest. Owicki and Lamport (cf. [9]) proposed the study of liveness properties in the context of concurrent programs. They were the first to to introduce the property $p$ leads-to $q$. In [2], Chandy and Misra show how properties like $p$ leads-to $q$ can be used to good advantage in the specification and design of programs. If $p$ leads-to $q$ is a property of the program, then this expresses the fact that if a state satisfying $p$ is encountered during execution of the program, it will eventually be followed by a state satisfying $q$. Such a property is called a progress property. (Here and in the remainder of this note, 'followed by' means that the second state coincides with the first, or comes later during execution of the program.)

A major difference in the semantic style between UNITY logic and the $w p$ calculus is that properties in UNITY logic are not calculated through equational reasoning but are derived from axioms and inference rules. Although $p$ and $q$ are predicates, $p$ leads-to $q$ is not a predicate: it is a property that may or may not be derivable. Constructs like $p$ leads-to ( $q$ leads-to $r$ ) are out of the question. In this note we propose predicate transformers for expressing progress. They relate the initial state with intermediate states and the final state. Because we take the final state into account, sequential composition fits in well. However, it turns out that we win and loose: although 
it is easy to deal with sequential composition, parallel composition is hard. In this note we do not discuss parallel composition at all.

We begin our study with an overview of results on predicates and predicate transformers as used in this context. It provides the notation and terminology for the remainder of this paper. Next, we link a proposed predicate transformer for expressing progress properties to an operational interpretation of program execution. This leads to a set of requirements that seem reasonable to expect from any predicate transformer that satisfies the interpretation. In the following section we define the predicate transformer by induction over the program structure, and we establish some theorems about it. Finally, we repeat these steps for another progress property and its predicate transformer.

\section{A summary of results in predicate calculus}

We use predicates to express conditions that may or may not hold in a certain state during program execution. Because of the state's omnipresence we would like it to be as anonymous as possible. Therefore, we have the following two conventions. If $e$ and $f$ are expressions and $\oplus$ is an operator of the appropriate type, $e \oplus f$ is also an expression. The value of $e \oplus f$ is in every point in the state space given by the value of $e$ in that point combined through $\oplus$ with the value of $f$ in the same point, i.e. operator $\oplus$ is applied pointwise. This is the usual interpretation for, say arithmetic operations, and we stick to the same rule for boolean operations. Hence, operators $\vee$ and $\wedge$ are applied pointwise, as usual, and so are $\equiv$ and $\Rightarrow$, which is unusual. The price that we have to pay for this convention is that $P \equiv Q$ does not express the fact that $P$ and $Q$ are the same predicates, i.e. that $P$ and $Q$ are boolean expressions whose values are pointwise equivalent, but it is again a predicate: a boolean expression that is true in those points where $P$ and $Q$ are equal, and false elsewhere. This is where the second convention comes in. We write $[P]$ to denote universal quantification of $P$ over all points in the state space. (Exactly what the state space is depends on the collection of program variables at hand and the advantage of the square brackets is that this collection can be left anonymous under the assumption that the collection of program variables is constant.) If $P$ is a predicate that is true in every point in the state space, $[P]$ is true, and $[P]$ is false for each predicate that is false in at least one point in the state space. Hence $[P \equiv Q]$ is true if $P$ and $Q$ are the same predicates, i.e. have the same value in every point in the state space, and false otherwise. This would traditionally be written as $P=Q$ and we avoid confusion by never applying $=$ to predicates.

As a result of our conventions, for arithmetic expressions $e$ and $f,(e+f) \cdot(e-f)$ is an arithmetic expression, $(e+f) \cdot(e-f)=e^{2}+f^{2}$ is a boolean expression that is true in those points in space where $f$ is 0 , and false elsewhere. Notice that $(e+f) \cdot(e-f)=e^{2}-f^{2}$ is a boolean expression that happens to be true in every point in space. Consequently $\left[(e+f) \cdot(e-f)=e^{2}-f^{2}\right]$ is the boolean true. Hence, we use the square brackets to express statements of fact.

Observe that without quantifications all our expressions would be pointwise. We know from experience that many interesting functions are not pointwise, such as the determinant of a matrix.

It turns out that we are often proving properties like $[A \Rightarrow C]$, for predicates $A$ and $C$ in a number of steps by showing for example $[A \equiv B]$ and $[B \Rightarrow C]$ for judiciously chosen intermediate predicate $B$. If the predicate $B$ is long, as is often the case in our proofs, we do not 
want to write it twice as $[A \equiv B]$ and $[B \Rightarrow C]$. In such a case we will write

$$
\begin{aligned}
& \text { A } \\
& \equiv{ }_{B} \quad\{\text { hint why }[A \equiv B]\} \\
& \Rightarrow \quad\{\text { hint why }[B \Rightarrow C]\}
\end{aligned}
$$

Besides implication and equivalence for connecting lines in a proof, we use the reverse of implication, called follows from and written $\Leftarrow$.

We also follow the convention introduced in [4] to write function application with an explicit period rather than implicitly, without a symbol. Hence, $f . x$ is function $f$ applied to argument $x$. Function application has a higher binding power than the other operators. The highest binding power of these is assigned to $\neg$, next come $\wedge$ and $\vee$, and finally $\equiv, \Rightarrow$, and $\Leftarrow$.

Quantified expressions mention the bound variables. For example $\langle\forall r: r \in R: q \vee r\rangle$ is a universal quantification over terms of the form $q \vee r$ where $q$ is a free variable and $r$ is a bound variable that ranges over all values in set $R$. If the range of $r$ is understood we abbreviate to $\langle\forall r:: q \vee r\rangle$.

For $f$ a predicate transformer, i.e. a function from predicates to predicates, we say that $f$ is monotonic if

$$
[p \Rightarrow q] \Rightarrow[f \cdot p \Rightarrow f \cdot q]
$$

for all predicates $p$ and $q$. We say that $f$ is universally conjunctive if

$$
[f .\langle\forall r: r \in R: r\rangle \equiv\langle\forall r: r \in R: f . r\rangle]
$$

holds for all sets of predicates $R$, independent of whether $R$ is finite, infinite, or empty. If the property holds for all nonempty sets $R$, we say that $f$ is positively conjunctive. Clearly, universal conjunctivity implies positive conjunctivity. We give a little theorem that will be used a lot. It shows that conjunctivity implies monotonicity.

\section{Theorem}

If $f$ is positively conjunctive then $f$ is monotonic.

Proof For all predicates $x$ and $y$,

$$
\begin{array}{ccc} 
& {[x \Rightarrow y]} \\
\equiv & & {[x \wedge y \equiv x]} \\
\Rightarrow & \{\text { Leibniz's rule }\} \\
& {[f .(x \wedge y) \equiv f . x]} \\
\equiv & \{\text { conjunctivity }\} \\
\equiv & {[f . x \wedge f . y \equiv f . x]} \\
& {[f . x \Rightarrow f . y]}
\end{array}
$$

(End of proof) 
In the proof we have referred to the rule of Leibniz. It is often paraphrased as "substitution of equals for equals". There is a dual theorem for disjunctive function, i.e. for functions that distribute over disjunction.

\section{Theorem}

If $f$ is positively disjunctive then $f$ is monotonic.

A "half-sided" counter-part to these theorems exists. For monotonic $f$, and any set $P$ of predicates,

$$
\begin{aligned}
& {[f .\langle\forall p: p \in P: p\rangle \Rightarrow\langle\forall p: p \in P: f . p\rangle] \quad \text { and }} \\
& {[f .\langle\exists p: p \in P: p\rangle \Leftarrow\langle\exists p: p \in P: f \cdot p\rangle] .}
\end{aligned}
$$

Sometimes, we have to solve equations in predicates. They are written in the form

$$
y: \text { [some predicate in } y \text { ] }
$$

and $y$ is a predicate variable. We say that $y$ is a solution if [some predicate in $y$ ] is true. Some equations have no solution, some have one solution, and others have more than one solution. Our equations generally fall in the latter category. They are often of the form

$$
y:[y \equiv f . y]
$$

for monotonic predicate transformer $f$. Sometimes we encounter

$$
y:[y \Leftarrow f . y]
$$

and its dual

$$
y:[y \Rightarrow f . y] \quad .
$$

Observe that (3) has at least one solution, viz. true, but in general it has more. Similarly, false is a solution of (4). For monotonic $f$, the conjunction of an arbitrary set of solutions of (3) is not necessarily a solution of (3). The following theorem shows, however, that the conjunction of all solutions is a solution. This conjunction $q$ is special in the sense that it is the strongest solution, i.e. $[q \Rightarrow z]$ for every solution $z$. If $f$ is not only monotonic but also universally conjunctive then the conjunction of any set of solutions is a solution.

\section{Theorem}

For monotonic $f$, equation (3) has a strongest solution, viz., the conjunction of all solutions of (3). Similarly, (4) has a weakest solution, viz., the disjunction of all solutions of (4).

Proof Let $S$ be the set of all solutions of equation (3), and let $q$ be their conjunction, i.e. $[q \equiv\langle\forall z: z \in S: z\rangle]$. We formulate $z \in S$ as $[z \Leftarrow f . z]$ and we prove $q \in S$ by showing $[q \Leftarrow f . q]$.

$$
\begin{aligned}
& f \cdot q \\
& \equiv \quad\{\text { definition of } q\} \\
& f .\langle\forall z:[z \Leftarrow f . z]: z\rangle \\
& \Rightarrow \quad\{f \text { is monotonic, }(0)\} \\
& \langle\forall z:[z \Leftarrow f . z]: f . z\rangle \\
& \Rightarrow \quad\{\text { quantification ranges only over } z \text { for which } f . z \text { implies } z\} \\
& \langle\forall z:[z \Leftarrow f . z]: z\rangle \\
& \equiv \quad\{\text { definition of } q\} \\
& q
\end{aligned}
$$


(End of proof)

Solutions to equations (2), (3), and (4) are related by the following important theorem, attributed to Knaster and Tarski (cf. [10]).

Theorem (Knaster-Tarski)

For monotonic f, equations (2) and (3) have the same strongest solution, and (2) and (4) have the same weakest solution.

Proof We restrict ourselves to (2) and (3). From the previous theorem we know that (3) has a strongest solution $q$, i.e. we have

$$
[f . z \Rightarrow z] \Rightarrow[q \Rightarrow z] \text { for all } z
$$

and

$$
[f . q \Rightarrow q] \quad \text {. }
$$

It remains to show that $q$ is a solution of (2), i.e. $[f . q \equiv q]$, and that $q$ is stronger than all other solutions, i.e. $[f . z \equiv z] \Rightarrow[q \Rightarrow z]$ for all $z$. We have

$$
\begin{aligned}
& {[q \Rightarrow z]} \\
& \Leftarrow \quad\{(5)\} \\
& {[f . z \Rightarrow z]} \\
& \Leftarrow \\
& {[f . z \equiv z]}
\end{aligned}
$$

and

$$
\begin{aligned}
& {[f \cdot q \equiv q]} \\
& \equiv \\
& {[f \cdot q \Rightarrow q] \wedge[f \cdot q \Leftarrow q]} \\
& \equiv \quad\{(6)\} \\
& {[f . q \Leftarrow q]} \\
& \Leftarrow \quad\{(5) \text { with } f . q \text { for } z\} \\
& {[f .(f . q) \Rightarrow f . q]} \\
& \Leftarrow \quad\{f \text { is monotonic }\} \\
& {[f . q \Rightarrow q]} \\
& \equiv \quad\{(6)\}
\end{aligned}
$$

(End of proof)

The function $f$ that figures in our equations usually depends on another predicate also, $z$ say. Therefore, we consider equation

$$
y:[y \equiv f . z . y] \quad .
$$

According to Knaster-Tarski, if $f$ is monotonic in $y,(7)$ has a strongest solution, $g . z$ say, and a weakest solution, h.z say. We mention some additional results (cf. [4]). If $f$ is universally or positively conjunctive in $(z, y), h$ is universally or positively conjunctive in $z$ respectively. If $f$ is universally or positively disjunctive in $(z, y), g$ is universally or positively disjunctive in $z$ respectively. Function $f$ is said to be universally conjunctive in $(z, y)$ if

$$
[\langle\forall z, y: z \in Z \wedge y \in Y: f . z . y\rangle \equiv f .\langle\forall z: z \in Z: z\rangle \cdot\langle\forall y: y \in Y: y\rangle]
$$


for all $Z$ and $Y$. The other definitions are similar. If $f$ is conjunctive, we have a property that relates $g$ and $h$. This property is crucial in the remainder.

$$
[g \cdot(x \wedge y) \equiv g \cdot x \wedge h \cdot y]
$$

In the cases that we encounter, f.z.y is of the form $z \wedge k . y$, where $z$ is independent of $y$, and $k$ is conjunctive. We may then rewrite equation (7) as

$$
y:[y \equiv z \wedge k \cdot y]
$$

We have the following property.

Theorem For any solution $x$ of (9),

$$
x \wedge h . z \text { is the weakest solution of } y:[y \equiv z \wedge k .(x \wedge y)] \quad \text {. }
$$

Proof First, we show that $x \wedge h . z$ solves the equation in (10).

$$
\begin{array}{cc} 
& x \wedge h . z \\
\equiv & \{x \text { and h.z solve }(9)\} \\
\equiv & z \wedge k . x \wedge z \wedge k .(h . z) \\
& \{\text { calculus }\} \\
\equiv & z \wedge k . x \wedge k .(h . z) \\
& z \wedge k .(x \wedge h . z)
\end{array}
$$

We rewrite the equation in (10) by observing

$$
\begin{array}{cc} 
& z \wedge k \cdot(x \wedge y) \\
\equiv & \{k \text { is conjunctive }\} \\
\equiv & z \wedge \cdot x \wedge k \cdot y \\
\equiv & \{k \cdot x \wedge z \wedge k \cdot y \\
& x \wedge z \wedge k \cdot y
\end{array}
$$

which yields equation

$$
y:[y \equiv x \wedge z \wedge k \cdot y]
$$

with weakest solution $h .(x \wedge z)$. It remains to show that the latter implies $x \wedge h . z$.

$$
\begin{array}{ll} 
& h .(x \wedge z) \\
\equiv \quad & \{h \text { is conjunctive }\} \\
& h . x \wedge h . z \\
\Rightarrow \quad & \{[h . x \equiv x \wedge k .(h . x)] \text { hence }[h . x \Rightarrow x]\}
\end{array}
$$

(End of proof)

\section{Semantics of programs through wlp and $w p$}

In this section we summarize the definitions of predicate transformers $w l p$ and $w p$ as given in $[3,4,5]$. The interpretation of $w l p$ and $w p$ is discussed in the next section; here we just list the 
definition and the requirements that are imposed on the definition of $w l p$ and $w p$ for all programs $s$ in order to admit a sensible interpretation. The two requirements, or healthiness conditions are (R0) and (R1).

wlp.s is universally conjunctive

$[$ wp.s.r $\equiv$ wp.s.true $\wedge$ wlp.s.r $]$ for all $r$

There is a third requirement, usually referred to as the Law of the Excluded Miracle,

$$
\text { [wp.s.false } \equiv \text { false }]
$$

but we have no use for it and ignore it in this paper. The definition of $w l p$ and $w p$ is by induction over the structure of the program.

$$
\begin{aligned}
& {[\text { wlp.skip.r } \equiv r]} \\
& {[\text { wp.skip.r } \equiv r]} \\
& {[w l p . a b o r t . r \equiv \text { true }]} \\
& {[w p . a b o r t . r \equiv \text { false }]} \\
& [w l p .(s ; t) . r \equiv \text { wlp.s.(wlp.t.r })] \\
& [w p \cdot(s ; t) . r \equiv \text { wp.s.(wp.t.r })] \\
& {\left[w l p .(x:=e) . r \equiv r_{e}^{x}\right]} \\
& {\left[w p .(x:=e) . r \equiv r_{e}^{x}\right]}
\end{aligned}
$$

For $I F=\mathbf{i f}\left\langle\llbracket i:: b_{i} \rightarrow s_{i}\right\rangle \mathbf{f i}$

$$
\begin{aligned}
& {\left[w l p . I F . r \equiv\left\langle\forall i: b_{i}: w l p . s_{i} . r\right\rangle\right]} \\
& {\left[w p . I F . r \equiv\left\langle\forall i: b_{i}: w p . s_{i} . r\right\rangle \wedge\left\langle\exists i:: b_{i}\right\rangle\right]}
\end{aligned}
$$

For $D O=$ do $b \rightarrow s$ od we obtain a defining equation by equating $D O$ and its first unfolding if $b \rightarrow s ; D O \llbracket \neg b \rightarrow$ skip fi. If the two are to be equal, the predicate transformers of $D O$ and its unfolding are to be equal also, i.e.

$$
\begin{aligned}
& {[w l p . D O . r \equiv(b \vee r) \wedge(\neg b \vee \text { wlp.s. }(w l p . D O . r))]} \\
& {[w p . D O . r \equiv(b \vee r) \wedge(\neg b \vee \text { wp.s. }(w p . D O . r))] .}
\end{aligned}
$$

We want to use the equations for defining the left-hand sides, but the two equations may have many solutions. Using (R1), the latter equation is equivalent to

$$
[w p . D O . r \equiv(b \vee r) \wedge(\neg b \vee \text { wp.s.true }) \wedge(\neg b \vee \text { wlp.s. }(w p . D O . r))] \quad .
$$

Abbreviating if $b \rightarrow s$ fi to $I F$, we see that wlp.DO.r and wp.DO.r are solutions of equations (11) and (12).

$$
\begin{aligned}
& y:[y \equiv(b \vee r) \wedge w l p . I F . y] \\
& y:[y \equiv(b \vee r) \wedge(\neg b \vee w p . s . t r u e) \wedge w l p . I F . y]
\end{aligned}
$$

Both (11) and (12) are of the form

$$
y:[y \equiv z \wedge w \operatorname{lp.IF.y}]
$$

and differ in the choice for $z$ only. Since $z \wedge w l p . I F . y$ is monotonic, (13) has a strongest solution $g . z$ and a weakest solution h.z. The predicate transformer wlp.DO is chosen to be the weakest 
solution of its defining equation, and wp.DO is chosen to be the strongest solution of its defining equation.

$$
\begin{aligned}
& {[w \operatorname{lp} . D O . r \equiv h .(b \vee r)]} \\
& {[w p . D O . r \equiv g .((b \vee r) \wedge(\neg b \vee w p . s . t r u e))]}
\end{aligned}
$$

The last line is (cf. (8)) equivalent to

$$
[w p . D O . r \equiv h .(b \vee r) \wedge g .(\neg b \vee \text { wp.s.true })] .
$$

The definitions of $w l p . D O$ and $w p . D O$ as given above are as in [4]. The definitions given in $[3,5]$ are different: they give a definition in terms of the limit of a sequence. The two definitions are equivalent if the predicate transformer of the repeated statement, i.e. wlp.s or wp.s, is continuous. (A predicate transformer $f$ is continuous if

$$
[\langle\exists p: p \in P: f \cdot p\rangle \equiv f \cdot\langle\exists p: p \in P: p\rangle]
$$

for every nonempty set of predicates $P$ that can be totally ordered into a sequence by implication.) If it is merely conjunctive the definitions are different and the limit need not solve the equations on which the present definitions are based. Restricting statements to continuous ones makes it hard, if not impossible, to construct programs by stepwise refinement, as argued in [0].

\section{An operational appreciation of predicate transformers}

In this section we introduce a classification of program executions. An execution of a program can be viewed as a sequence of states; the actual sequence depends on the program text and on implementation choices. One classification is based on termination: execution does or does not terminate. In the latter case, we say that we have an eternal execution. Executions that terminate do so in some final state, and they are further distinguished by whether or not they satisfy some given condition on the final state. The condition is expressed as a predicate $r$ on the program's state space, and each state satisfies either $r$ or $\neg r$. The $w l p$ and $w p$ calculus is based on partitioning the executions of a (fixed) program $s$ into three classes:

eternal : the executions that do not terminate,

finally $r \quad$ : the executions that terminate in a final state satisfying $r$,

finally $\neg r \quad$ : the executions that terminate in a final state satisfying $\neg r$.

Every execution falls into exactly one of these three classes. Predicate transformers $w l p$ and $w p$ can be defined in terms of these classes. For program s, predicates wlp.s.r and wp.s.r are as follows.

wlp.s.r : holds in those initial states for which no execution of $s$ belongs to the class 'finally $\neg r$

wp.s.r : holds in those initial states for which no execution of $s$ belongs to the class 'eternal' or to the class 'finally $\neg r$ '

Predicate transformer $w l p$ corresponds to what is called partial correctness whereas $w p$ corresponds to what is called total correctness. Usually, $w p$ is used in the specification of programs, whereas $w l p$ has more attractive mathematical properties. The two are related through

$$
[\text { wp.s.r } \equiv \text { wp.s.true } \wedge \text { wlp.s.r }] \quad .
$$


In this paper we propose a different characterization by considering a predicate $q$ and partition executions in two classes, depending on whether $q$ holds at some state. We consider all states: the initial state, the final state (if any), and all states in between.

ever $q \quad$ : the executions such that $q$ holds in some state

never $q \quad$ : the executions such that $q$ holds in no state

Observe that this partitioning is independent of the previous one: an execution in 'ever $q$ ' can be in 'eternal', 'finally $r$ ', or in 'finally $\neg r$ ', and so can an execution in 'ever $\neg q$ '. Of course, an execution in 'finally $q$ ' is in 'ever $q$ '.

As indicated before, we aim at a predicate transformer that maps predicate $q$ to a predicate on the initial state (the precondition) such that an execution is in 'ever $q$ ' if the precondition is satisfied. Since we anticipate sequential composition of programs, and since the state in which $q$ holds during execution of $(s ; t)$ may, loosely speaking, be either a state of $s$ or of $t$, we are also interested in the state in which $s$ terminates. For a fixed program we, therefore, partition program executions into four classes.

ever $q$

never $q$ and eternal

never $q$ and finally $r$

never $q$ and finally $\neg r$
: the executions such that $q$ holds in some state

: the executions such that $q$ holds in no state and that do not terminate

: the executions such that $q$ holds in no state and that terminate in a final state satisfying $r$

: the executions such that $q$ holds in no state and that terminate in a final state satisfying $\neg r$.

For given $s, q$, and $r$, this classification leads us to define two predicates.

wlev.s.q.r : holds in those initial states for which no execution of $s$ belongs to the class "never $q$ and finally $\neg r$,

wev.s.q.r : holds in those initial states for which no execution of $s$ belongs to the class "never $q$ and eternal' or to the class 'never $q$ and finally $\neg r$ '

The name wev is derived from 'weakest ever' and is chosen to reflect the property that wev.s.q.r is the weakest condition on the initial state such that execution of $s$ is guaranteed to ever visit a state that satisfies $q$ or to terminate in a state that satisfies $r$. The $l$ in wlev stands for wlev being the liberal version of wev. In a later section we discuss an even more involved predicate transformer that expresses properties like 'a state in which $p$ holds is eventually followed by a state in which $q$ holds'. We postpone the introduction thereof until after we have studied wlev and wev.

\section{Requirements on predicate transformers}

Not all predicate transformers can meaningfully be interpreted as a wlev.s or as a wev.s for some program s. We discuss a number of requirements that are motivated by operational concerns. When we define wlev and wev for a variety of statements in the next section, we check that these requirements are met.

If we choose for $q$ the predicate false, the class 'ever $q$ ' is empty and the partitioning into the remaining three classes coincides with the partitioning underlying the definition of wlp.s and 
wp.s. Hence, we require that substitution of false for $q$ reduces wlev and wev to wlp and wp respectively.

$$
\begin{aligned}
& \text { [wlev.s.false.r } \equiv \text { wlp.s.r }] \\
& {[\text { wev.s.false.r } \equiv \text { wp.s.r }]}
\end{aligned}
$$

Since $w l p$ is universally conjunctive, and because of (E0), we expect a similar conjunctive property for wlev. What exactly should the requirement be? Let $R$ be any (possible empty) set of predicates. We have

no execution belongs to the class 'never $q$ and finally $\neg\langle\forall r: r \in R: r\rangle$ '

$$
\equiv
$$

$$
\langle\forall r: r \in R \text { : no execution belongs to the class 'never } q \text { and finally } \neg r \text { ' }\rangle
$$

and conclude that we need to require universal conjunctivity of wlev : for each $q$ and each program $s$

$$
\text { wlev.s.q is universally conjunctive . }
$$

A consequence of (E1) is (take the conjunction of zero predicates)

$$
\text { [wlev.s.q.true] . }
$$

We have already mentioned formula (R1), which expresses the relation between $w l p$ and $w p$. Since $w l p$ and $w p$ are in turn related to wlev and wev we expect a similar relation between the latter two. We have

wev.s.q.true : holds in those initial states for which no execution of $s$ belongs to the class "never $q$ and eternal'

and conclude that, for each program $s$, and all predicates $q$ and $r$, we need to require

$$
\text { [wev.s.q.r } \equiv \text { wev.s.q.true } \wedge \text { wlev.s.q.r }] \quad \text {. }
$$

On account of (17) both left- and right-hand side of (E2) reduce to wev.s.q.true. From (E1) and (E2) it follows that for each program $s$, and all predicates $q$ and $r$, we have

$$
\text { wev.s.q is positively conjunctive . }
$$

So much for the properties that were inspired by wlp and $w p$. The remaining three properties are peculiar to wlev and wev. If a state satisfies some predicate $q$, it also satisfies every weaker predicate $q^{\prime}$, and therefore every execution in class 'ever $q$ ' is also in the larger class 'ever $q$ '. As a result we expect wev.s.q.r and wlev.s.q.r to be monotonic in q. This leads to our next requirements.

$$
\begin{aligned}
& {\left[q \Rightarrow q^{\prime}\right] \Rightarrow\left[\text { wlev.s.q.r } \Rightarrow \text { wlev.s. } q^{\prime} . r\right]} \\
& {\left[q \Rightarrow q^{\prime}\right] \Rightarrow\left[\text { wev.s.q.r } \Rightarrow \text { wev.s.q. } q^{\prime} . r\right]}
\end{aligned}
$$

Observe that neither of (E3) and (E3'), combined with (E2), implies the other one. Finally we consider the fact that the initial and final states of an execution are also intermediate states. This suggests the requirements

$$
\begin{aligned}
& {[\text { wlev.s.q.r } \equiv \text { wlev.s.q. }(q \vee r)]} \\
& {[q \Rightarrow \text { wev.s.q.r }]}
\end{aligned}
$$

which in combination with (E2) imply 


$$
\begin{aligned}
& {[\text { wev.s.q.r } \equiv \text { wev.s.q. }(q \vee r)]} \\
& {[q \Rightarrow \text { wlev.s.q.r }]}
\end{aligned}
$$

This completes our list of requirements. We conclude this section with a little theorem. It expresses that we can strengthen the postcondition of a wlev with that of a wlp. If either the wlev or wlp is replaced by its nonliberal counterpart, the conclusion is nonliberal.

\section{Theorem}

For all programs $s$, and predicates $q, r$, and $w$, we have

$$
\begin{aligned}
& {[\text { wlev.s.q.r } \wedge \text { wlp.s.w } \Rightarrow \text { wlev.s.q. }(r \wedge w)]} \\
& {[\text { wlev.s.q. } r \wedge \text { wp.s.w } \Rightarrow \text { wev.s.q. }(r \wedge w)]} \\
& {[\text { wev.s.q.r } \wedge \text { wlp.s.w } \Rightarrow \text { wev.s.q. }(r \wedge w)]} \\
& {[\text { wev.s.q.r } \wedge \text { wp.s.w } \Rightarrow \text { wev.s.q. }(r \wedge w)]}
\end{aligned}
$$

Proof We give the proof for (18a); the other three are similar.

$$
\begin{array}{ccc} 
& & \text { wlev.s.q. } r \wedge \text { wlp.s.w } \\
\equiv & & \{(\mathrm{E} 0)\} \\
& \text { wlev.s.q. } r \wedge \text { wlev.s.false.w } \\
\Rightarrow & \{(\mathrm{E} 3)\} \\
& \text { wlev.s.q.r } \wedge \text { wlev.s.q.w } \\
\equiv & \{(\mathrm{E} 1)\} \\
& \text { wlev.s.q. }(r \wedge w)
\end{array}
$$

(End of proof)

One might be tempted to replace the implications in this theorem by equivalences. The example

$$
\begin{gathered}
s=\text { if true } \rightarrow \cdots\{q\} \cdots\{r \wedge \neg w\} \\
\quad \text { \true } \rightarrow \cdots\{r \wedge w\} \\
\quad \mathbf{f i}
\end{gathered}
$$

(in which $\cdots$ stands for a terminating statement that establishes the condition following it) shows that such temptations had better be resisted since

$$
\begin{aligned}
& {[\text { wlev.s.q. }(r \wedge w) \equiv \text { true }]} \\
& {[\text { wlev.s.q.r } \equiv \text { true }]} \\
& {[w \text { lp.s.q.w } \equiv \text { false }]}
\end{aligned}
$$

We have a long list of requirements on wlev and wev and one wonders whether they are independent. Here we give a number of functions that meet each but one of the requirements for wlev. A similar list can be compiled for wev. For wlev.s.q.r that meets all requirements except one, choose

all except (E0) : true

all except (E1) : wlp.s. $(q \vee r) \vee q \vee(\neg[\neg q] \wedge \neg r)$

all except (E2) : not applicable, because we consider wlev only

all except (E3) $: q \quad \vee \quad(([\neg q] \vee r) \wedge w l p . s .(q \vee r))$ 
all except (E4) : $q \vee$ wlp.s.r

all except $(\mathrm{E} 5) \quad: \quad$ lp.s. $(q \vee r)$

We omit the proof that, for example, the choice $q \vee$ wlp.s.r satisfies (E0) through (E5). The most complicated case is wlp.s. $(q \vee r) \vee q \vee(\neg[\neg q] \wedge \neg r)$ which meets all requirements except (E1), the conjunctivity. An example is: substitute $x:=4$ for $s$, false for $q$, and $\{$ false, $x=3\}$ for the set of predicates $R$.

One might also wonder whether our requirements capture all properties of wlev and wev. This is not the case. For example, assume that one has an implementation that executes a program without any intermediate states, i.e., it proceeds in one step from initial to final state. The requirements that we have discussed do not address this issue at all. Therefore, one may expect that the pair consisting of $q \vee w l p . s .(q \vee r)$ and $q \vee w p . s .(q \vee r)$ satisfies all requirements. Fortunately, it does. In the next section we discuss a definition of wlev and wev by induction on the structure of programs. The definitions admit the traditional operational interpretation of programs (cf. [4]) in which, for example, an intermediate state exists between execution of $s$ and $t$ in $(s ; t)$.

\section{Definition of wlev and wev}

In this section we give definitions of wlev and wev by induction on the structure of the programs. For each construct we have to verify that requirements (E0), (E0'), (E1), (E2), (E3), (E3'), (E4), and (E5') are met. Most proofs are omitted; only the proofs for the case of the iterative command are listed in the appendix.

We begin our list of definitions with perhaps the simplest statement of all: skip, the statement that does not change the state whatsoever. The definition of skip is

$$
\begin{aligned}
& {[\text { wlev.skip.q.r } \equiv q \vee r]} \\
& {[\text { wev.skip.q.r } \equiv q \vee r] .}
\end{aligned}
$$

The second statement that we define is abort. It is the statement that has an initial state but no other intermediate state and has no final state. Or, equivalently, it repeats the initial state indefinitely. This suggests the following definition.

$$
\begin{aligned}
& {[\text { wlev.abort.q.r } \equiv \text { true }]} \\
& {[\text { wev.abort.q.r } \equiv q]}
\end{aligned}
$$

The next construct that we define is sequential composition. The execution of $s$; $t$ is the execution of $s$, followed by execution of $t$ if $s$ terminates. Hence, we are led to define

$$
\begin{aligned}
& {[\text { wlev.(s;t).q.r } \equiv \text { wlev.s.q.(wlev.t.q.r)] }} \\
& [\text { wev.(s;t).q.r } \equiv \text { wev.s.q.(wev.t.q.r })] .
\end{aligned}
$$

The simplicity of these two definitions was one of our motivations for introducing predicate transformers that operate on two predicates in the first place.

(Intermezzo) This is the point where the second predicate, $r$, plays its role. We tried to avoid $r$ and defined wev.s.q to be the weakest precondition such that execution of $s$ would ever visit a state in which $q$ holds. The problem is that a definition like

$$
[\text { wev.(s;t).q } \equiv \text { wev.s.q } \vee \text { wp.s. }(\text { wev.t.q })]
$$


is inappropriate because of possible nondeterminism in $s$.

We show that a few properties that one might expect on operational grounds are indeed theorems. First we show that skip is the left and right unit element of sequential composition. Next we show that abort is the left zero element, and finally we show that sequential composition is associative.

\section{Theorem}

For all programs $s$ we have

$$
\text { s;skip }=s=\text { skip } ; s .
$$

Proof Equality of programs depends on the context: it is with respect to the properties that one looks at. In our present context, equality of programs means that their predicate transformers wlev and wev are equal. It follows from (E0) and (E0') that wlp and $w p$ are special cases of wlev and wev. Therefore, equality of programs with respect to wlev and wev implies equality with respect to $w l p$ and $w p$. We give the proof for equality with respect to wev only; the proof for wlev is similar. For all $s, q$, and $r$ we have

$$
\begin{aligned}
& \text { wev. }(s ; s k i p) . q . r \\
& \equiv \quad\{\text { definition ; }\} \\
& \text { wev.s.q.(wev.skip.q.r) } \\
& \equiv \quad\{\text { definition skip }\} \\
& \text { wev.s.q. }(q \vee r) \\
& \equiv \quad\left\{\left(\mathrm{E} 4^{\circ}\right)\right\} \\
& \text { wev.s.q.r } \\
& \equiv \quad\left\{\left(\mathrm{E} 5^{\circ}\right)\right\} \\
& q \vee \text { wev.s.q.r } \\
& \equiv \quad\{\text { definition skip }\} \\
& \text { wev.skip.q.(wev.s.q.r) } \\
& \equiv \quad\{\text { definition ; }\} \\
& \text { wev.(skip;s).q.r . }
\end{aligned}
$$

(End of proof)

\section{Theorem}

For all programs $s$ we have

abort; $s=$ abort

Proof We give the proof for equality with respect to wev only; the proof for wlev is similar. For all $s, q$, and $r$ we have

$$
\begin{aligned}
& \text { wev.(abort; s).q.r } \\
& \equiv \quad\{\text { definition ; }\} \\
& \text { wev.abort.q.(wev.s.q.r) } \\
& \equiv \quad\{\text { definition abort }\} \\
& q \\
& \equiv \quad\{\text { definition abort }\} \\
& \text { wev.abort.q.r. }
\end{aligned}
$$

(End of proof) 
In general, abort is not the right zero element of sequential composition. If a state is reached by execution of $s$ from some initial state then that same state is reached by executing $s$; abort. Hence, wev.(s;abort).q.r is weaker, holds in more initial states, than wev.abort.q.r does.

\section{Theorem}

Sequential composition is associative.

Proof We give the proof for associativity with respect to wev only; the proof for wlev is similar. For all $s, t, u, q$, and $r$ we have

$$
\begin{array}{cc} 
& \text { wev. }(s ;(t ; u)) \cdot q . r \\
\equiv & \{\text { definition ; }\} \\
& \text { wev.s.q. }(\text { wev. }(t ; u) \cdot q . r) \\
\equiv & \{\text { definition ; }\} \\
& \text { wev.s.q.(wev.t.q.(wev.u.q.r })) \\
\equiv & \{\text { definition ; }\} \\
& \text { wev.(s;t).q.(wev.u.q.r) } \\
\equiv & \{\text { definition ; }\} \\
& \text { wev. }((s ; t) ; u) \cdot q . r
\end{array}
$$

(End of proof)

We continue our list of definitions and tackle the only statement that changes the state: the assignment statement. We consider the assignment $x:=e$ and ignore the concurrent assignment. The definition uses a function dom. We have dom.e = true if the evaluation of $e$ is within its domain, and dom.e $=$ false otherwise. For example, dom. $(1 / x)=(x \neq 0)$. The operational interpretation of the assignment $x:=e$ reveals that there are two states: one before and one after the assignment. They differ in the value for the $x$ coordinate only.

$$
\begin{aligned}
& \text { wlev. }(x:=e) . q . r \equiv q \vee\left(\neg \text { dom.e cor }\left(q_{e}^{x} \vee r_{e}^{x}\right)\right) \\
& \text { wev. }(x:=e) . q . r \equiv q \vee\left(\text { dom.e cand }\left(q_{e}^{x} \vee r_{e}^{x}\right)\right)
\end{aligned}
$$

Usually we ignore the possibility of an expression being evaluated outside its domain, in which case we can simplify the two formulae by replacing dom.e with true.

$$
\begin{aligned}
& {\left[w \operatorname{lev} \cdot(x:=e) \cdot q \cdot r \equiv q \vee q_{e}^{x} \vee r_{e}^{x}\right]} \\
& {\left[w e v \cdot(x:=e) \cdot q \cdot r \equiv q \vee q_{e}^{x} \vee r_{e}^{x}\right]}
\end{aligned}
$$

In the sequel we stick to the latter two.

\section{Example}

We give an example to show that programs that are equal with respect to $w l p$ and $w p$ semantics can be different with respect to wlev and wev semantics. The two programs that we compare are $i:=3 ; j:=4$ and $j:=4 ; i:=3$. Since for both programs wp.s.r and wlp.s.r is $r_{3,4}^{i, j}$ they are 
indistinguishable with respect to $w l p$ and $w p$. However, we have

$$
\begin{array}{ll} 
& \text { wev. }(i:=3 ; j:=4) .(i>j) \cdot f a l s e \\
\equiv \quad & \text { wev. }(i:=3) .(i>j) .(\text { wev } \cdot(j:=4) .(i>j) . f a l s e) \\
\equiv \quad & \text { wev. }(i:=3) \cdot(i>j) .(i>j \vee i>4) \\
\equiv \quad & i>j \vee 3>j \vee 3>j \vee 3>4 \\
\equiv \quad & i>j \vee 3>j
\end{array}
$$

and

$$
\begin{aligned}
& \text { wev. }(j:=4 ; i:=3) .(i>j) . \text { false } \\
& \equiv \quad \text { wev. }(j:=4) .(i>j) .(\text { wev. }(i:=3) .(i>j) . \text { false }) \\
& \equiv \\
& \text { wev. }(j:=4) .(i>j) .(i>j \vee 3>j) \\
& \equiv \\
& i>j \vee i>4 \vee i>4 \vee 3>4 \\
& \equiv \\
& i>j \vee i>4
\end{aligned}
$$

which are distinct.

(End of example)

The last statement but one that we consider is the alternative statement. Abbreviated to IF, the alternative statement is written as a list of guarded commands, joined by vertical bars, [, and surrounded by the bracket pair if $\mathrm{fi}$. We write $b_{i} \rightarrow s_{i}$ for a guarded command, and assume the range for $i$ to be understood.

$$
\begin{aligned}
& {\left[\text { wlev.IF.q.r } \equiv\left\langle\forall i: b_{i}: \text { wlev.s. } s_{i} . q . r\right\rangle\right]} \\
& {\left[\text { wev.IF.q.r } \equiv\left\langle\forall i: b_{i}: \text { wev.s. } s_{i} . q . r\right\rangle \wedge\left(q \vee\left\langle\exists i:: b_{i}\right\rangle\right)\right]}
\end{aligned}
$$

The last, and more challenging statement to define is the repetition. We look at the case of a repetition consisting of one guarded command only. At the expense of more notational inconvenience it can readily be generalized to more (or fewer) guarded commands. We use the abbreviation $D O$ for the repetition with guard $b$ and body $s$.

$$
D O=\operatorname{do} b \rightarrow s \text { od }
$$

We shall find it convenient to have IF stand for the same statement in which the outer bracket pair is replace by that of an alternative statement

$$
I F=\text { if } b \rightarrow s \mathbf{f i}
$$

with the same $b$ and $s$. The definition of wlev.DO and wev.DO is inspired by the operational interpretation of $D O$. It begins by stipulating that $D O$ equals its first 'unfolding'

$$
D O=\text { if } b \rightarrow s ; D O \llbracket \neg b \rightarrow \operatorname{skip} \mathbf{f i}
$$


which suggests

$$
\begin{aligned}
& {[\text { wlev.DO.q.r } \equiv(\neg b \vee \text { wlev. }(s ; D O) . q . r) \wedge(b \vee \text { wlev.skip.q.r })]} \\
& {[\text { wev.DO.q.r } \equiv(\neg b \vee \text { wev. }(s ; D O) . q . r) \wedge(b \vee \text { wev.skip.q.r })]}
\end{aligned} .
$$

By substituting the definitions of ; and skip we can simplify these definitions to the following two.

$$
\begin{aligned}
& {[\text { wlev.DO.q.r } \equiv(\neg b \vee \text { wlev.s.q. }(\text { wlev.DO.q. } r)) \wedge(b \vee q \vee r)]} \\
& {[\text { wev.DO.q.r } \equiv(\neg b \vee \text { wev.s. }(\text { wev.DO.q.r })) \wedge(b \vee q \vee r)]}
\end{aligned}
$$

This is the easy part. The harder part is formed by the observation that, when viewed as an equation in the unknown wlev.DO.q.r and wev.DO.q.r, these equations may, for given $b$ and $s$, have more than one solution. We have to specify which solutions we want to be the definitions of wlev. DO and wev.DO. We have a closer look at the two equations. We replace the unknown quantities by a fresh variable $y$ to make the character of the equations more explicit. We find that wlev.DO.q.r is a solution of equation

$$
y:[y \equiv(\neg b \vee \text { wev.s.q.y }) \wedge(b \vee q \vee r)]
$$

in $y$, and that wev.DO.q.r is a solution of equation

$$
y:[y \equiv(\neg b \vee \text { wev.s.q.y }) \wedge(b \vee q \vee r)] \quad .
$$

Applying (E2) to the last equation we obtain

$$
y:[y \equiv(\neg b \vee \text { wev.s.q.true }) \wedge(\neg b \vee \text { wlev.s.q.y }) \wedge(b \vee q \vee r)] \quad .
$$

We rewrite the equations in terms of wlev.IF.



$$
\begin{aligned}
& y:[y \equiv \text { wlev.IF.q.y } \wedge(\neg b \vee \text { wev.s.q.true }) \wedge(b \vee q \vee r)]
\end{aligned}
$$

Observe that both equations are of the form

$$
y:[y \equiv z \wedge \text { wlev.IF.q.y }] \quad,
$$

differing in the choice for $z$ only. ( $z$ depends on $b, s$, $q$, and $r$, but not on $y$.) Therefore, we concentrate on equation (23). As a function of $y, z \wedge$ wlev.IF.q.y is positively conjunctive and, hence, monotonic. According to Knaster-Tarski's theorem this implies that (23) has a strongest solution, g.z say, and a weakest solution, h.z say. Since $z \wedge$ wlev.IF.q.y is universally conjunctive in $(z, y)$, we have

$$
[g . z \equiv g . t r u e \wedge h . z] \quad .
$$

Furthermore, $h$ is universally conjunctive.

Next, we give an operational interpretation of g.true and h.z in order to motivate our choices for wlev.DO and wev.DO. Observe that g.true is the strongest solution of

$$
y:[y \equiv \text { wlev.IF.q.y }] \quad .
$$

Let $c$ be any solution of this equation. An execution started in a state satisfying $c$ is started in a state in which wlev.IF.q.c, i.e. $\neg b \vee$ wev.s.q.c, holds. If $\neg b$ execution of the loop terminates; if wlev.s.q.c execution of $s$ is in the class 'eternal', or in 'ever $q$ ', or in 'finally $c$ '. By induction, we find that an execution of $D O$ started in a state satisfying $c$ is in one of four classes: the iteration stops after a finite number of steps in a state satisfying $c \wedge \neg b$, or one of the executions of $s$ visits a state in which $q$ holds, or after a finite number of iterations the loop goes into an execution of 
$s$ that does not terminate, or the loop repeats $s$ forever. These are the four possibilities for an arbitrary solution of (24). Next we show that the last of these four possibilities is ruled out if $c$ happens to be the strongest solution of (24). Let us define $d$ to be the predicate that holds precisely in those initial states such that, after a finite number of executions of $s, D O$ terminates or another execution of $s$ is started which is in the class 'ever $q$ ' or in the class 'eternal'.

From the definition of $d$ we have $[\neg b \Rightarrow d]$ because initial state $\neg b$ implies immediate termination of $D O$, which is a possibility included in $d$. We also have $[$ wlev.s.q.d $\Rightarrow d]$ because initial state wlev.s.q.d implies that execution of $s$ visits $q$, or terminates in $q$, or does not terminate at all; each of these three is included in $d$. Together they imply that $d$ is a solution of $y:[y \Leftarrow \neg b \vee$ wlev.s.q.y $]$, i.e. of

$$
y:[y \Leftarrow \text { wlev.IF.q.y }] \text {. }
$$

From Knaster-Tarski's theorem, we know that equations (24) and (25) have the same strongest solution. From the definition of $d$ we conclude $[d \Rightarrow c]$, for every solution $c$ of (24), including the strongest solution g.true of (24). Since g.true is also the strongest solution of (25), we find that $d$ is a solution of (25), and it is at least as strong as the strongest solution of (25). This all goes to say that $d$ is the strongest solution of (25), i.e. $d \equiv g$.true. (How did the infinite repetition of $s$ disappear? It is handled by Knaster-Tarski's theorem, simple as it may appear to be.)

Next we consider, for fixed $z, h . z$, the weakest solution of

$y:[y \equiv z \wedge$ wlev.IF.q.y $] \quad$.

This turns out to be a hard job, the hard part being the interaction between $q$ and $z$, and we make it a bit easier by restricting ourselves to predicates $z$ that are implied by $q$, i.e. we assume $[q \Rightarrow z]$. Define $e$ to be the predicate that holds exactly in those initial states of $D O$ for which $z$ holds prior to every iteration of the loop as long as $q$ has not been true.

From the definition of $e$ we have that, if execution of $D O$ is started in a state that satisfies $e$, either $\neg b$ holds and the loop terminates, or $b$ holds and leads to an execution of $s$ that either does not terminate, or visits $q$, or terminates in a state in which $e$ holds again. This amounts to the implication $[e \Rightarrow \neg b \vee$ wlev.s.q.e]. Furthermore, if $e$ holds initially then also either $q$ or $z$ holds. Since $[q \Rightarrow z]$ we conclude that $[e \Rightarrow z]$. (By the way, this was the reason for restricting our attention to $[q \Rightarrow z]$.) Combined with the other implication, we find that e satisfies $[e \Rightarrow z \wedge(\neg b \vee$ wlev.s.q.e $)]$ which simplifies to $[e \Rightarrow z \wedge$ wlev.IF.q.e]. This shows that $e$ is a solution of equation (26).

$$
y:[y \Rightarrow z \wedge \text { wlev.IF.q.y }]
$$

According to Knaster-Tarski's theorem, equations (26) and (23) have the same weakest solution h.z. Since $e$ is a solution of (26), it implies the weakest solution.

$$
[e \Rightarrow h . z]
$$

Let $f$ be an arbitrary solution of (26). An execution of DO starting a state that satisfies $f$ either terminates right away or initiates an execution of $I F$ that -because $f$ is a solution of (26) - does not terminate, or visits $q$, or terminates in $f$. Hence, unless $q$ has been true, prior to each iteration $f$ holds. From (26) we conclude that $f$ implies $z$ and, unless $q$ has been true, prior to each iteration $z$ holds as well. From the definition of $e$ it follows that $f$ implies e. Since this conclusion holds for every $f$ that solves (26) it also holds for the weakest solution $h . z$.

$$
[h . z \Rightarrow e]
$$


The combination of (27) and (28) shows that h.z equals $e$, which provides the characterization of $h . z$ that we were looking for (but only for those $z$ that satisfy $[q \Rightarrow z]$ !). Since $[q \Rightarrow b \vee q \vee r]$ we may substitute (cf. (21)) $b \vee q \vee r$ for $z$ in the interpretation of $h . z$ and find that $h .(b \vee q \vee r)$ holds exactly in those initial states of $D O$ for which $b \vee q \vee r$ holds prior to every iteration of the loop, as long as $q$ has not been true. In other words: $h .(b \vee q \vee r)$ holds exactly in those initial states of $D O$ such that no execution is in the class 'never $q$ and finally $\neg r$ '.

Since $[q \Rightarrow \neg b \vee$ wev.s.q.true $]$ we may substitute (cf. (22)) $\neg b \vee$ wev.s.q.true for $z$ in the interpretation of $h . z$ and find that $h .(\neg b \vee$ wev.s.q.true $)$ holds exactly in those initial states of $D O$ for which $\neg b \vee$ wev.s.q.true holds prior to every iteration of the loop, as long as $q$ has not been true. Together with g.true we have: g.true $\wedge$ h. $(\neg b \vee$ wev.s.q.true $)$ holds exactly in those initial states of $D O$ such that no execution is in the class "never $q$ and eternal'.

From this lengthy detour through equations in predicates we find our definitions for wlev.DO.q.r and wev.DO.q.r.

$$
\begin{aligned}
& {[\text { wlev.DO.q.r } \equiv h .(b \vee q \vee r)]} \\
& {[\text { wev.DO.q.r } \equiv \text { g.true } \wedge \text { h. }(\neg b \vee \text { wev.s.q.true }) \wedge h .(b \vee q \vee r)]}
\end{aligned}
$$

The latter can, thanks to $g \cdot(x \wedge y) \equiv g . x \wedge h . y$, be simplified to

$$
[\text { wev.DO.q.r } \equiv g \cdot((\neg b \vee \text { wev.s.q.true }) \wedge(b \vee q \vee r))]
$$

We have shown that they satisfy equations (21) and (22) that were derived from the first unfolding of $D O$ and we have shown that they agree with the operational interpretation. In the appendix we show that they satisfy the seven requirements that we have imposed earlier. We now show that skip and abort are two extreme cases of the DO-statement.

\section{Theorem}

For each statement $s$,

$$
\text { skip }=\text { do false } \rightarrow \text { s od }
$$

Proof We give the proof of equality with respect to wlev only; the proof for wev is similar. We have

$$
\begin{aligned}
& \text { wlev.(do false } \rightarrow \text { s od).q.r } \\
& \equiv \quad\{\text { definition } D O\} \\
& \text { the weakest solution of } y:[y \equiv \text { wlev.(if false } \rightarrow s \mathbf{f i}) . q . y \wedge(\text { false } \vee q \vee r)] \\
& \equiv \quad\{\text { definition IF }\} \\
& \text { the weakest solution of } y:[y \equiv \text { true } \wedge(\text { false } \vee q \vee r)] \\
& \equiv \quad\{\text { calculus }\} \\
& \text { the weakest solution of } y:[y \equiv q \vee r] \\
& \equiv \quad\{\text { definition of weakest solution }\} \\
& \equiv \quad q \vee r \\
& \text { wlev.skip.q.r }
\end{aligned}
$$

(End of proof)

\section{Theorem}

$$
\text { abort }=\text { do true } \rightarrow \text { skip od }
$$


Proof Again, we give the proof of equality with respect to wlev only; the proof for wev is similar. We have

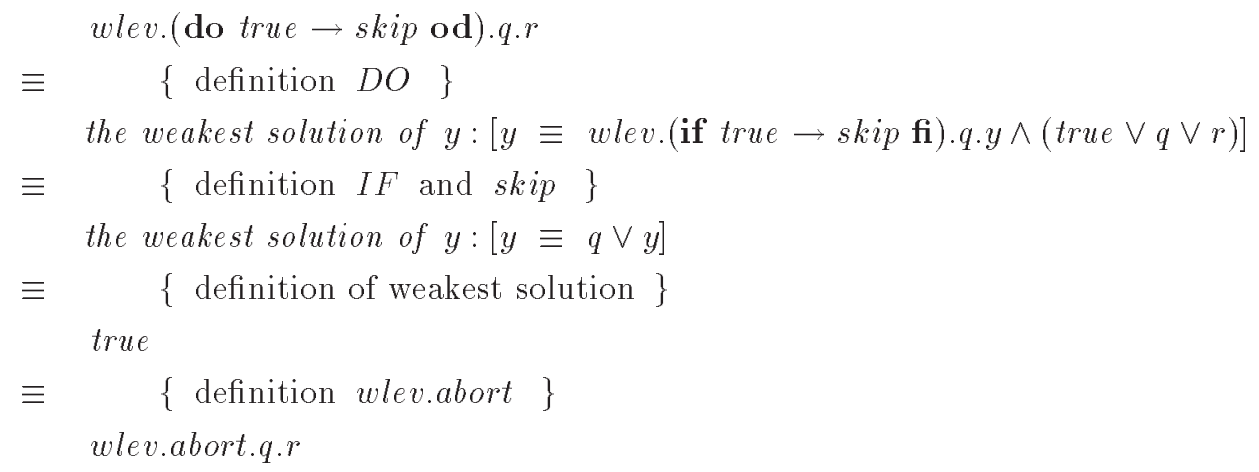

(End of proof)

Next we present an invariance theorem for wev. It is a generalization of the invariance theorem for $w p$.

\section{Theorem}

Consider the program

$$
D O=\operatorname{do} b \rightarrow s \text { od } .
$$

Let $(D, \leq)$ be a partially ordered set and let $C$ be a subset of $D$ such that $(C, \leq)$ is well founded.

Let $I, q$, and $r$ be predicates on the state and $t$ an expression of type $D$ such that

$$
\begin{aligned}
& {[I \wedge b \Rightarrow t \in C]} \\
& \langle\forall x: x \in C:[I \wedge b \wedge t=x \Rightarrow \text { wev.s.q. }(I \wedge(b \vee q \vee r) \wedge t<x)]\rangle ;
\end{aligned}
$$

then

$$
[I \wedge(b \vee q \vee r) \Rightarrow \text { wev.DO.q.r }] \quad .
$$

Before we give the proof, we show that this theorem is a true generalization of the theorem for $w p$. With no great surprise, we substitute false for $q$ and $I \wedge \neg b$ for $r$ and find: if

$$
[I \wedge b \Rightarrow t \in C]
$$

and

$$
\langle\forall x: x \in C:[I \wedge b \wedge t=x \Rightarrow \text { wp.s. }(I \wedge t<x)]\rangle
$$

then

$$
[I \Rightarrow \text { wp.DO. }(I \wedge \neg b)] \quad .
$$

This is exactly the familiar invariance theorem for $w p$.

Observe that the replacement of $r$ by $I \wedge \neg b$ is something that we could have done in our theorem and proof also. We have chosen not to do so because that would allow us to weaken the postcondition easily, but does not permit strengthening it. And when proving progress properties that is exactly what we often do: we strengthen the postcondition $r$ to false and the 'true' progress condition $q$ remains.

Proof The proof that we give is similar to the proof for $w p$ 's invariance theorem as found in [4]. If we let $w$ stand for wev.DO.q.r then $w$ is the strongest solution of equation 
JAN $163 \mathrm{a}-19$

$$
[y \equiv f . y]
$$

where $[f . y \equiv(b \vee q \vee r) \wedge(\neg b \vee$ wev.s.q.y $)]$. In fact, we show that the invariance theorem holds for any solution $w$ of (35).

Our proof obligation (34) is $[I \wedge(b \vee q \vee r) \Rightarrow w]$. From requirement (E5') and the definition of $f$ we have, for any $e$,

$$
[q \Rightarrow f . e]
$$

which in conjunction with $[w \equiv f . w]$ implies $[q \Rightarrow w]$ thereby reducing our proof obligation to $[I \wedge(b \vee r) \Rightarrow w]$. Since

$$
[I \wedge(b \vee r) \Rightarrow w] \equiv[I \wedge(b \vee r) \wedge t \in C \Rightarrow w] \wedge[I \wedge(b \vee r) \wedge t \notin C \Rightarrow w]
$$

and

$$
\begin{aligned}
& {[I \wedge(b \vee r) \wedge t \notin C \Rightarrow w]} \\
& \equiv \quad\{[b \vee r \equiv b \vee(\neg b \wedge r)]\} \\
& {[I \wedge b \wedge t \notin C \Rightarrow w] \wedge[I \wedge \neg b \wedge r \wedge t \notin C \Rightarrow w]} \\
& \equiv \quad\{(32)\} \\
& {[I \wedge \neg b \wedge r \wedge t \notin C \Rightarrow w]} \\
& \equiv \quad\{w \text { is a solution of }(35)\} \\
& {[I \wedge \neg b \wedge r \wedge t \notin C \Rightarrow f . w]} \\
& \equiv \quad\{\text { definition of } f\} \\
& {[I \wedge \neg b \wedge r \wedge t \notin C \Rightarrow(b \vee q \vee r) \wedge(\neg b \vee \text { wev.s.q.w })]} \\
& \equiv \quad\{\text { calculus }\} \\
& \text { true }
\end{aligned}
$$

we have

$$
[I \wedge(b \vee r) \Rightarrow w] \equiv[I \wedge(b \vee r) \wedge t \in C \Rightarrow w]
$$

and our proof obligation reduces to

$$
[I \wedge(b \vee r) \wedge t \in C \Rightarrow w] \quad .
$$

Rewriting it even further we obtain

$$
\begin{aligned}
& {[I \wedge(b \vee r) \wedge t \in C \Rightarrow w]} \\
& \equiv \\
& {[\langle\forall x: t=x: I \wedge(b \vee r) \wedge x \in C \Rightarrow w\rangle]} \\
& \equiv \\
& {[\langle\forall x: x \in C: I \wedge(b \vee r) \wedge t=x \Rightarrow w\rangle]} \\
& \equiv \\
& \langle\forall x: x \in C:[I \wedge(b \vee r) \wedge t=x \Rightarrow w]\rangle
\end{aligned}
$$

and we prove the latter by mathematical induction, i.e. we prove it under the assumption

$$
\langle\forall y: y \in C \wedge y<x:[I \wedge(b \vee r) \wedge t=y \Rightarrow w]) \quad .
$$


In order to apply (33), we rewrite it in terms of $f$ first. For $x \in C$ we have

$$
\begin{array}{ll} 
& I \wedge b \wedge t=x \Rightarrow \text { wev.s.q. }(I \wedge(b \vee r) \wedge t<x) \\
\equiv \quad & I \wedge b \wedge t=x \Rightarrow b \wedge \text { wev.s.q. }(I \wedge(b \vee r) \wedge t<x) \\
\equiv \quad & I \wedge(b \vee q \vee r) \wedge(b \vee \neg(q \vee r)) \wedge t=x \Rightarrow b \wedge \text { wev.s.q. }(I \wedge(b \vee r) \wedge t<x) \\
\equiv \quad & \quad I \wedge(b \vee q \vee r) \wedge t=x \Rightarrow(\neg b \wedge(q \vee r)) \vee(b \wedge \text { wev.s.q. }(I \wedge(b \vee r) \wedge t<x)) \\
\equiv \quad & \quad\{(36)\} \\
\equiv \quad & I \wedge(b \vee q \vee r) \wedge t=x \Rightarrow f .(I \wedge(b \vee r) \wedge t<x) \\
\equiv & I \wedge(b \vee r) \wedge t=x \Rightarrow f .(I \wedge(b \vee r) \wedge t<x)
\end{array}
$$

so that we can rewrite (33) as

$$
\langle\forall x: x \in C:[I \wedge(b \vee r) \wedge t=x \Rightarrow f .(I \wedge(b \vee r) \wedge t<x)]\rangle
$$

and proceed with the proof.

$$
\begin{array}{ccc} 
& \langle\forall y: y \in C \wedge y<x:[I \wedge(b \vee r) \wedge t=y \Rightarrow w]\rangle \\
\equiv & {[\langle\forall y: y \in C \wedge y<x: I \wedge(b \vee r) \wedge t=y \Rightarrow w\rangle]} \\
\equiv \quad & {[\langle\forall y: t=y \wedge y<x: I \wedge(b \vee r) \wedge y \in C \Rightarrow w\rangle]} \\
\equiv \quad & \quad[I \wedge(b \vee r) \wedge t<x \wedge t \in C \Rightarrow w] \\
\equiv \quad & \quad\{(37)\} \\
& {[I \wedge(b \vee r) \wedge t<x \Rightarrow w]} \\
\Rightarrow \quad & \quad\{f \text { is monotonic }\} \\
& {[f .(I \wedge(b \vee r) \wedge t<x) \Rightarrow f . w]} \\
\Rightarrow \quad & \quad\{(38)\} \\
& {[(I \wedge(b \vee r) \wedge t=x) \Rightarrow f . w]} \\
\equiv \quad & \{w \text { is a solution of }(35)\} \\
& {[(I \wedge(b \vee r) \wedge t=x) \Rightarrow w]}
\end{array}
$$

(End of proof)

We conclude this section with an example. Consider the following program.

$$
S=i:=10 ; \text { do } i \neq 0 \rightarrow i:=i-1 \text { od }
$$

We want to prove [wev.S. $(i=3)$.false], i.e. during execution of $S$ a state is encountered during which $i$ equals 3 . We guess that an appeal to the invariance theorem is required with

$$
\begin{aligned}
& I: \quad 3 \leq i \\
& t: \quad i \\
& C: \quad \text { natural numbers }
\end{aligned}
$$


and we boldly go ahead, pretending that we can work out the invariance later.

$$
\begin{aligned}
& \text { wev.S. }(i=3) \text {.false } \\
& \equiv \quad\{\text { definition of } S\} \\
& \text { wev. }(i:=10) .(i=3) .(\text { wev.DO. }(i=3) . \text { false }) \\
& \Leftarrow \quad\{[I \wedge(i \neq 0 \vee i=3) \Rightarrow \text { wev.DO. }(i=3) . \text { false }] \text {, see below; (E1') }\} \\
& \text { wev. }(i:=10) .(i=3) .(I \wedge(i \neq 0 \vee i=3)) \\
& \equiv \quad\{[I \wedge(i \neq 0 \vee i=3) \equiv 3 \leq i]\} \\
& \text { wev. }(i:=10) .(i=3) .(3 \leq i) \\
& \equiv \quad\{\text { definition of assignment }\} \\
& i=3 \vee 10=3 \vee 3 \leq 10 \\
& \equiv \\
& \text { true }
\end{aligned}
$$

It remains to check $[I \wedge(i \neq 0 \vee i=3) \Rightarrow$ wev.DO. $(i=3)$.false $]$, which we do next. By the invariance theorem, this boils down to checking

$$
[I \wedge i \neq 0 \Rightarrow i \geq 0]
$$

which is immediate from the definition of $I$, and checking

$$
\langle\forall x: x \geq 0:[I \wedge i \neq 0 \wedge i=x \Rightarrow \text { wev. }(i:=i-1) .(i=3) .(I \wedge(i \neq 0 \vee i=3) \wedge i<x)]\rangle
$$

which follows from

$$
\begin{array}{ll} 
& \text { wev. }(i:=i-1) \cdot(i=3) .(I \wedge(i \neq 0 \vee i=3) \wedge i<x) \\
\equiv \quad & \text { wev. }(i:=i-1) \cdot(i=3) .(3 \leq i<x) \\
\equiv \quad & i=3 \vee i-1=3 \vee 3 \leq i-1<x) \\
\equiv \quad & \quad i=3 \vee i=4 \vee 4 \leq i \leq x \\
\Leftarrow \quad & 3 \leq i \wedge i=x \\
\equiv \quad & I \wedge i \neq 0 \wedge i=x
\end{array}
$$

for all $x$.

\section{An exploration of leads-to}

As mentioned before, one of our main sources of inspiration is the progress property $p$ leads-to $q$ as used in UNITY. The property $[I \Rightarrow$ wev.s.q.false] expresses that execution of $s$ from an initial state that satisfies $I$ will lead to a state that satisfies $q$. It is more restricted than leads - to in the sense that one predicate is restricted to the initial state. In this section we explore a method for expressing something like leads - to as a predicate transformer. Similar to what we have done before, we partition executions of a program into classes. Instead of partitioning them into 'ever $q$ ' 
and 'never $q$ ' we use two predicates, $p$ and $q$, and the partitioning is in two classes, depending on whether in an execution every state in which $p$ holds is eventually followed by a state in which $q$ holds.

$p$ leads to $q$ : the executions such that every state in which $p$ holds is eventually followed by a state in which $q$ holds

$p$ without $q$ : the executions such that there is a state in which $p$ holds which is not followed by a state in which $q$ holds

Observe that the executions in which no state satisfies $p$ are in ' $p$ leads to $q$ '. Observe also that the class ' true leads to $q$ ' is a proper subset of the class 'ever $q$ '. As before we anticipate sequential composition, i.e. we anticipate interest in the final state, and distinguish four classes.

$p$ leads to $q \quad$ : the executions such that every state in which $p$ holds is eventually followed by a state in which $q$ holds

$p$ without $q$ and eternal : the executions such that there is a state in which $p$ holds which is not followed by a state in which $q$ holds and that do not terminate

$p$ without $q$ and finally $r$ : the executions such that there is a state in which $p$ holds which is not followed by a state in which $q$ holds and that terminate in a state satisfying $r$

$p$ without $q$ and finally $\neg r$ : the executions such that there is a state in which $p$ holds which is not followed by a state in which $q$ holds and that terminate in a state satisfying $\neg r$

For given $s, p, q$, and $r$, this classification leads us to define two predicates.

wlto.s.p.q.r : holds in those initial states for which no execution belongs to the class ' $p$ without $q$ and finally $\neg r$ '

wto.s.p.q.r

: holds in those initial states for which no execution belongs to the class ' $p$ without $q$ and finally $\neg r$ ' or to the class ' $p$ without $q$ and eternal'

As before, not all predicate transformers can meaningfully be interpreted as a wlto.s or a wto.s for some program $s$, and we discuss a number of requirements inspired by operational interpretations. Again, we choose constants for some of the parameters to obtain wlp and $w p$ as special cases of wlto and wto respectively. Substituting true for $p$ and false for $q$ we obtain

$$
\begin{aligned}
& {[\text { wlto.s.true.false.r } \equiv \text { wlp.s.r }]} \\
& {[\text { wto.s.true.false.r } \equiv \text { wp.s.r }] .}
\end{aligned}
$$

However, a stronger result is possible for wlto. According to the operational interpretation, wlto.s.p.q. $(\neg p \vee$ $q \vee r$ ) holds exactly in those initial states for which no execution is in the class ' $p$ without $q$ and finally $p \wedge \neg q \wedge \neg r^{\prime}$. If an execution terminates in a state satisfying $p \wedge \neg q \wedge \neg r$, then $p$ holds but there is no way to establish either $q$ or $r$. Hence wlto.s.p.q. $(\neg p \vee q \vee r)$ holds exactly in those initial states for which no execution is in the class 'finally $p \wedge \neg q \wedge \neg r$ '. Consequently

$$
[\text { wlto.s.p.q. }(\neg p \vee q \vee r) \equiv \text { wlp.s. }(\neg p \vee q \vee r)] \quad .
$$

For wto and $w p$ we see no way to strengthen (T0'). 
Since wlp is conjunctive, and because of (T0), we expect a conjunctivity property for wlto. We require, for all $s, p$, and $q$,

wlto.s.p.q is universally conjunctive

which implies the special case (the conjunction of zero predicates)

$$
\text { [wlto.s.p.q.true] . }
$$

We have come to expect a relation between the liberal and the conservative predicate transformer. They are coupled by substituting true for the the last parameter of the conservative function.

$$
\text { [wto.s.p.q.r } \equiv \text { wto.s.p.q.true } \wedge \text { wlto.s.p.q.r] }
$$

Thanks to (39) both left- and right-hand side of (T2) reduce to wto.s.p.q.true when substituting true for $r$. From (T1) and (T2) it follows that, for all $s, p$, and $q$,

wto.s.p.q is positively conjunctive .

So much for the properties related to the $r$ argument, Next we turn to the second argument, viz. q. Just like in the case of wlev and wev we require monotonicity of the predicate transformers as a function of $q$, and, again, the requirements are independent for the two functions.

$$
\begin{aligned}
& {\left[q \Rightarrow q^{\prime}\right] \Rightarrow\left[\text { wlto.s.p.q.r } \Rightarrow \text { wlto.s.p.q. } q^{\prime} \cdot r\right]} \\
& {\left[q \Rightarrow q^{\prime}\right] \Rightarrow\left[\text { wto.s.p.q.r } \Rightarrow \text { wto.s.p.q. } q^{\prime} \cdot r\right]}
\end{aligned}
$$

Properties (E4) and (E4') express that the final state is also an intermediate state. The pendant for leads - to is similar. We have

$$
[\text { wlto.s.p.q.r } \equiv \text { wlto.s.p.q. }(q \vee r)]
$$

which implies

$$
[w t o . s . p . q . r \equiv w t o . s . p . q .(q \vee r)]
$$

Properties (E5) and (E5') express that the initial state is also an intermediate state. The pendant for leads - to is that a state satisfying $p$ is also a state that coincides with or follows a state satisfying $p$.

$$
\text { [wto.s.p.p.r] }
$$

In combination with the monotonicity in $q$ this can be phrased as

$$
[p \Rightarrow q] \Rightarrow[\text { wto.s.p.q.r }] \quad \text {. }
$$

In combination with (T2), (T5') implies

$$
[\text { wlto.s.p.p.r] }
$$

and

$$
[p \Rightarrow q] \Rightarrow[\text { wlto.s.p.q.r }] \quad \text {. }
$$

This brings us to consider the first predicative argument, $p$. Suppose a computation is in the class ' $p 0$ leads to $q$ or terminates in $r$ ' and also in the class ' $p 1$ leads to $q$ or terminates in $r$ '. Then it is also in the class ' $p 0 \vee p 1$ leads to $q$ or terminates in $r$ ', and vice versa. We extend this observation to the 'junctivity' requirements (T6) and (T6'). For each, possibly empty, set of predicates $P$ we require

$$
\begin{aligned}
& {[\langle\forall p: p \in P: \text { wlto.s.p.q.r }\rangle \equiv \text { wlto.s. }\langle\exists p: p \in P: p\rangle \cdot q . r] \quad ;} \\
& {[\langle\forall p: p \in P: \text { wto.s.p.q.r }\rangle \equiv \text { wto.s. }\langle\exists p: p \in P: p\rangle \cdot q . r]}
\end{aligned}
$$


The remaining two pairs of requirements capture important 'progress' characteristics of our predicate transformers. The first pair expresses that an execution that is in the class 'ever $p$ ' and in the class ' $p$ leads to $q$ ' is also in the class 'ever $q$ '.

$$
\begin{aligned}
& \text { [wlto.s.p.q.r } \wedge \text { wlev.s.p.r } \Rightarrow \text { wlev.s.q.r] } \\
& {[\text { wto.s.p.q.r } \wedge \text { wev.s.p.r } \Rightarrow \text { wev.s.q.r }]}
\end{aligned}
$$

The second and last pair expresses transitivity: an execution that is in the class ' $p$ leads to $q$ ' and in the class ' $q$ leads to $w$ ' is also in the class ' $p$ leads to $w$ '.

$$
\begin{aligned}
& \text { [wlto.s.p.q.r } \wedge \text { wlto.s.q.w.r } \Rightarrow \text { wlto.s.p.w.r] } \\
& {[\text { wto.s.p.q.r } \wedge \text { wto.s.q.w.r } \Rightarrow \text { wto.s.p.w.r] }}
\end{aligned}
$$

This completes our list of requirements for wlto and wto. Since they have three predicates as arguments instead of two, we are not surprised that the list is longer than for wlev and wev. The next section defines wlto and wto for a number of statements. this section concludes with a

theorem that is similar to a theorem about wlev and wev, and we have a theorem on monotonicity in the $p$ argument.

\section{Theorem}

For all programs $s$, and predicates $p, q, r$, and $w$, we have

$$
\begin{aligned}
& {[\text { wlto.s.p.q. } r \wedge \text { wlp.s.w } \Rightarrow \text { wlto.s.p.q. }(r \wedge w)]} \\
& {[\text { wlto.s.p.q. } r \wedge \text { wp.s.w } \Rightarrow \text { wto.s.p.q. }(r \wedge w)]} \\
& {[\text { wto.s.p.q. } r \wedge \text { wlp.s.w } \Rightarrow \text { wto.s.p.q. }(r \wedge w)]} \\
& {[\text { wto.s.p.q. } r \wedge \text { wp.s.w } \Rightarrow \text { wto.s.p.q. }(r \wedge w)]}
\end{aligned}
$$

Proof We give the proof for (40a); the other three are similar.

$$
\begin{aligned}
& \text { wlto.s.p.q.r } \wedge \text { wlp.s.w } \\
& \equiv \quad\{(\mathrm{T} 0)\} \\
& \text { wlto.s.p.q.r } \wedge \text { wlto.s.true.false.w } \\
& \Rightarrow \quad\{(\mathrm{T} 3) \text { and }(\mathrm{T} 6)\} \\
& \text { wlto.s.p.q.r } \wedge \text { wlto.s.p.q.w } \\
& \equiv \quad\{(\mathrm{T} 1)\} \\
& \text { wlto.s.p.q. }(r \wedge w)
\end{aligned}
$$

(End of proof)

\section{Theorem}

For all programs $s$, and predicates $p, p^{\prime}, q, r$, and $w$, we have

$$
\begin{aligned}
& {\left[p^{\prime} \Rightarrow p\right] \Rightarrow[\text { wlto.s.p.q.r } \Rightarrow \text { wlto.s.p. } . q . r]} \\
& {\left[p^{\prime} \Rightarrow p\right] \Rightarrow\left[\text { wto.s.p.q.r } \Rightarrow \text { wto.s.p. } p^{\prime} . q . r\right]}
\end{aligned}
$$

Proof We give the proof for (41a); the other one is similar. Assume $\left[p^{\prime} \Rightarrow p\right]$.

$$
\begin{array}{cc} 
& \text { wlto.s.p.q.r } \wedge \text { wlto.s.p'.q.r } \\
& \{(\mathrm{T} 6)\} \\
& \text { wlto.s. }\left(p \vee p^{\prime}\right) \cdot q \cdot r \\
& \text { wlto.s.p.q.r }
\end{array}
$$


(End of proof)

\section{Definition of wlto and wto}

In this section we give the definitions of wlto and wto by induction on the structure of the programs. For each construct we have to verify that the requirements are met. Again, most proofs are omitted; only the proofs for the case of the iterative command are listed in the appendix.

We begin our list of definitions with skip, the statement that does not change the state whatsoever. The definition of skip is

$$
\begin{aligned}
& {[\text { wlto.skip.p.q.r } \equiv \neg p \vee q \vee r]} \\
& {[\text { wto.skip.p.q.r } \equiv \neg p \vee q \vee r] .}
\end{aligned}
$$

The second statement that we define is abort. It is the statement that has an initial state but no other intermediate state and has no final state. This suggests the following definition.

$$
\begin{aligned}
& {[\text { wlto.abort.p.q.r } \equiv \text { true }]} \\
& {[\text { wto.abort.p.q.r } \equiv \neg p \vee q]}
\end{aligned}
$$

The next construct that we define is sequential composition. The execution of $s ; t$ is the execution of $s$, followed by execution of $t$ if $s$ terminates. If $p$ leads to $q$ in $s$; $t$ then a state satisfying $p$ that is encountered during execution of $s$ leads to a state satisfying $q$ that is encountered during $s$ or else $s$ terminates in a state from which $t$ is started and that leads to a state satisfying $q$. Furthermore, if $s$ terminates, then it does so in a state in which $p$ leads to $q$ during execution of $t$. Hence, we are suggested to define

$$
\begin{aligned}
& [w l t o .(s ; t) . p . q . r \equiv \text { wlto.s.p.q.(wlev.t.q.r }) \wedge \text { wlp.s.(wlto.t.p.q.r })] \\
& [w t o .(s ; t) \cdot p \cdot q . r \equiv \text { wto.s.p.q.(wev.t.q.r }) \wedge \text { wlp.s.(wto.t.p.q.r })] \quad .
\end{aligned}
$$

We show that a few properties that one might expect on operational grounds are indeed theorems. First we show that skip is the left and right unit element of sequential composition. Next we show that abort is the left zero element, and finally we show that sequential composition is associative.

\section{Theorem}

For all programs $s$ we have

$$
\text { s;skip }=s=\text { skip;s }
$$

Proof The equality of programs means that their predicate transformers wlto and wto are equal. Observe that, since $w l p$ and $w p$ are special cases of wlto and wto, equality of programs with respect to wlto and wto implies equality with respect to $w l p$ and $w p$. It does not imply equality with respect to wlev and wev, however. We give the proof for equality with respect to wlto only; 
the proof for wto is similar. For all $s, p, q$, and $r$ we have

$$
\begin{aligned}
& \text { wlto. }(\text { s; skip).p.q.r } \\
& \equiv \quad\{\text { definition ; }\} \\
& \text { wlto.s.p.q.(wlev.skip.q.r }) \wedge \text { wlp.s.(wlto.skip.p.q.r }) \\
& \equiv \quad\{\text { definition skip }\} \\
& \text { wlto.s.p.q. }(q \vee r) \wedge w l p . s .(\neg p \vee q \vee r) \\
& \equiv \quad\{(\mathrm{T} 0)\} \\
& \text { wlto.s.p.q. }(q \vee r) \wedge w l t o . s . p . q .(\neg p \vee q \vee r) \\
& \equiv \quad\{(\mathrm{T} 1)\} \\
& \text { wlto.s.p.q. }(q \vee r) \\
& \equiv \quad\{(\mathrm{T} 4)\} \\
& \text { wlto.s.p.q.r } \\
& \equiv \quad\{\text { see below }\} \\
& (\neg p \vee \text { wlev.s.q.r }) \wedge \text { wlto.s.p.q.r } \\
& \equiv \quad\{(\mathrm{E} 5)\} \\
& (\neg p \vee q \vee \text { wlev.s.q.r }) \wedge \text { wlto.s.p.q.r } \\
& \equiv \quad\{\text { definition skip }\} \\
& \text { wlto.skip.p.q.(wlev.s.q.r }) \wedge \text { wlp.skip.(wlto.s.p.q.r }) \\
& \equiv \quad\{\text { definition ; }\} \\
& \text { wlto.(skip;s).p.q.r }
\end{aligned}
$$

in which we have used the fact that

$$
\begin{array}{ll} 
& {[\text { wlto.s.p.q.r } \Rightarrow \neg p \vee \text { wlev.s.q.r }]} \\
\equiv \quad & \{\text { calculus }\} \\
& {[\text { wlto.s.p.q.r } \wedge p \Rightarrow \text { wlev.s.q.r }]} \\
\Leftarrow \quad & \{(\mathrm{E} 5)\} \\
\equiv & {[\text { wlto.s.p.q.r } \wedge \text { wlev.s.p.r } \Rightarrow \text { wlev.s.q.r }]} \\
& \{(\mathrm{T} 7)\}
\end{array}
$$

(End of proof)

\section{Theorem}

For all programs $s$ we have

abort;s $=$ abort

Proof We give the proof for equality with respect to wto only; the proof for wlto is similar. For all $s, q$, and $r$ we have

$$
\begin{aligned}
& w t o .(a b o r t ; s) \cdot p \cdot q \cdot r \\
& \equiv \quad\{\text { definition ; }\} \\
& \text { wto.abort.p.q.(wto.s.q.r) }) \text { wlp.abort.(wto.s.p.q.r }) \\
& \equiv \quad\{\text { definition abort }\} \\
& \neg p \vee q \\
& \equiv \quad \begin{array}{r}
\text { definition abort }\} \\
\text { wto.abort.p.q.r }
\end{array}
\end{aligned}
$$


(End of proof)

\section{Theorem}

Sequential composition is associative.

Proof We give the proof for associativity with respect to wto only; the proof for wlto is similar. For all $s, t, u, q$, and $r$ we have

$$
\begin{aligned}
& \text { wto. }(s ;(t ; u)) \cdot p . q . r \\
& \equiv \quad\{\text { definition ; }\} \\
& \text { wto.s.p.q.(wev.t.q.(wev.u.q.r)) } \\
& \wedge \text { wlp.s. }(\text { wto.t.p.q.(wev.u.q.r }) \wedge \text { wlp.t. }(\text { wto.u.p.q.r })) \\
& \equiv \quad\{\text { conjunctivity of wlp }\} \\
& \text { wto.s.p.q.(wev.t.q.(wev.u.q.r)) } \\
& \wedge \text { wlp.s.(wto.t.p.q.(wev.u.q.r })) \wedge \text { wlp.s. }(\text { wlp.t. }(\text { wto.u.p.q.r })) \\
& \equiv \quad\{\text { definition ; }\} \\
& \text { wto. }(s ; t) . p . q .(w e v . u . q . r) \wedge w l p .(s ; t) .(w t o . u . p . q . r) \\
& \equiv \quad\{\text { definition ; }\} \\
& \text { wto. }((s ; t) ; u) \cdot p \cdot q . r \quad \text {. }
\end{aligned}
$$

(End of proof)

Next, we consider the assignment $x:=e$. We ignore the possibility of an expression being evaluated outside its domain. The definitions are obtained by considering the fact that there are two states in the execution of the assignment statement: one before and one after the assignment. If $p$ leads to $q$, and $p$ holds initially then either $q$ holds also or $q \vee r$ holds in the final state; if $p$ holds in the final state then $q \vee r$ holds also.

$$
\begin{aligned}
& {\left[w \text { lto. }(x:=e) \cdot p \cdot q . r \equiv\left(\neg p \vee q \vee q_{e}^{x} \vee r_{e}^{x}\right) \wedge\left(\neg p_{e}^{x} \vee q_{e}^{x} \vee r_{e}^{x}\right)\right]} \\
& {\left[w t o .(x:=e) \cdot p \cdot q . r \equiv\left(\neg p \vee q \vee q_{e}^{x} \vee r_{e}^{x}\right) \wedge\left(\neg p_{e}^{x} \vee q_{e}^{x} \vee r_{e}^{x}\right)\right]}
\end{aligned}
$$

The last statement but one that we consider is the alternative statement. Abbreviated to IF, the alternative statement is written as a list of guarded commands, joined by vertical bars, [, and surrounded by the bracket pair if $\mathrm{fi}$. We write $b_{i} \rightarrow s_{i}$ for a guarded command, and assume the range for $i$ to be understood.

$$
\begin{aligned}
& {\left[\text { wlto.IF.p.q.r } \equiv\left\langle\forall i: b_{i}: \text { wlto.s.s.p.q.r }\right\rangle\right]} \\
& {\left[w \text { to.IF.p.q.r } \equiv\left\langle\forall i: b_{i}: \text { wto.s } s_{i} \cdot p . q . r\right\rangle \wedge\left(\neg p \vee q \vee\left\langle\exists i:: b_{i}\right\rangle\right)\right]}
\end{aligned}
$$

The last, and more challenging statement to define is the repetition. We use the abbreviation $D O$ for the repetition with guard $b$ and body $s$.

$$
D O=\operatorname{do} b \rightarrow s \text { od }
$$

Just as in the previous section, we find it convenient to have IF stand for the same statement in which the outer bracket pair is replace by that of an alternative statement

$$
I F=\text { if } b \rightarrow s \mathbf{f i}
$$


with the same $b$ and $s$. The definition of wlto.DO and wto.DO is inspired by its operational interpretation. Equating $D O$ and its first 'unfolding'

$$
D O=\text { if } b \rightarrow s ; D O \llbracket \neg b \rightarrow \operatorname{skip} \mathbf{f i}
$$

suggests

$$
\begin{aligned}
& {[\text { wlto.DO.p.q.r } \equiv(\neg b \vee \text { wlto. }(s ; D O) . p . q . r) \wedge(b \vee \text { wlto.skip.p.q.r })]} \\
& {[w \text { to.DO.p.q.r } \equiv(\neg b \vee w t o .(s ; D O) . p . q . r) \wedge(b \vee \text { wto.skip.p.q.r })]}
\end{aligned}
$$

By substituting the definitions of ; and skip we can simplify these definitions. Replacing the unknown quantities by a fresh variable $y$ to make the character of the equation more explicit we find that wlto.DO.p.q.r is a solution of

$$
y:[y \equiv(\neg b \vee(\text { wlp.s.y } \wedge \text { wlto.s.p.q. }(\text { wlev.DO.q.r }))) \wedge(b \vee \neg p \vee q \vee r)]
$$

and that wto.DO.p.q.r is a solution of the similar equation (43).

$$
y:[y \equiv(\neg b \vee(\text { wlp.s.y } \wedge \text { wto.s.p.q. }(\text { wev.DO.q.r }))) \wedge(b \vee \neg p \vee q \vee r)]
$$

Both equations are of the form

$$
y:[y \equiv z \wedge w l p . I F . y] \quad,
$$

differing in the choice for $z$ only. Remember that we have studied the similar equation (23) earlier. In (23) we have wlev.IF.q.y instead of wlp.IF.y. Therefore, equations (23) and (44) are equal if we substitute false for $q$ (cf. (E0)). Since equation (44) equals equation (13), the $g$ and $h$ that we have now are the same as the $g$ and $h$ that we have in the context of wlp.DO and wp.DO. In order to see which solution we have to choose as the appropriate one, we argue as follows. We claim that wto.DO.p.q.r is the weakest solution of (43). Let $y$ be any solution of (43). It suffices to show that $y$ implies wto.DO.p.q.r. Assume that $y$ is a precondition of the loop. From the conjunct $\neg b \vee$ wlp.s.y in (43), it follows that $y$ is an invariant of the loop. Whenever $p$ holds in some intermediate state of command $s$, predicate $b \wedge y$ holds at the beginning of that command and, hence, also wto.s.p.q.(wev.DO.q.r). This implies that $s$ establishes, after the state in which $p$ holds, an intermediate state in which $q$ holds or that $s$ terminates in a state in which wev.DO.q.r holds. In the latter case it follows that the remainder of the execution of the loop reaches an intermediate state in which $q$ holds or terminates in a state in which $r$ holds. Consequently, the initial state satisfies wto.DO.p.q.r. A similar reasoning applies to wlto.DO.p.q.r. We are thus led to the following definitions.

$$
\begin{aligned}
& \text { [wlto.DO.p.q.r } \equiv \text { h. }((b \vee \neg p \vee q \vee r) \wedge(\neg b \vee \text { wlto.s.p.q.(wlev.DO.q.r }))] \\
& {[w t o . D O . p . q . r \equiv h .((b \vee \neg p \vee q \vee r) \wedge(\neg b \vee \text { wto.s.p.q.(wev.DO.q.r }))]}
\end{aligned}
$$

Observe that both wlto.DO and wto.DO have been defined as the weakest solution of an equation, not as a weakest and a strongest solution respectively. This is our main reason for extensively including the operational interpretation of the predicates. In an earlier attempt we defined wto as the strongest solution, and were unable to prove one of the requirements (T5'). One might say that the strongest solution restricts the loop to a finite number of iterations, whereas the operational interpretation of wto.DO does not. The weakest solution does not limit the number of iterations either. We mention two extreme cases of the $D O$-statement. The proofs are omitted.

\section{Theorem}


For each statement $s$,

$$
\text { skip }=\text { do false } \rightarrow s \text { od }
$$

\section{Theorem}

$$
\text { abort }=\text { do true } \rightarrow \text { skip od }
$$

We conclude this section with an invariance theorem for wto. It is somewhat different from the invariance theorems for wev and $w p$ in the sense that there is no variant function, and all 'real' work is delegated to the wev of the same loop.

\section{Theorem}

Consider program $D O=$ do $b \rightarrow s$ od. We have

$$
[J \wedge b \Rightarrow \text { wto.s.p.q. }(w e v . D O . q . r) \wedge w l p . s .(J \wedge(b \vee \neg p \vee q \vee r))]
$$

implies

$$
[J \wedge(b \vee \neg p \vee q \vee r) \Rightarrow w t o . D O \cdot p \cdot q . r] \quad .
$$

Proof The weakest solution h.z of $y:[y \equiv f . z . y]$ is, for monotonic $f$, characterized by

$$
[x \Rightarrow f . z . x] \Rightarrow[x \Rightarrow h . z] \quad \text {. }
$$

Since wto.DO.p.q.r is the weakest solution of (43), it suffices to show $[x \Rightarrow f . z . x]$, where

$$
\begin{aligned}
& {[x \equiv J \wedge(b \vee \neg p \vee q \vee r)]} \\
& {[f . z . x \equiv w l p . I F . x \wedge(\neg b \vee w t o . s . p . q .(w e v . D O . q . r)) \wedge(b \vee \neg p \vee q \vee r)]} \\
& I F=\text { if } b \rightarrow s \mathbf{f i} .
\end{aligned}
$$

Here we go.

$$
\begin{aligned}
& {[x \Rightarrow f . z . x]} \\
& \equiv \quad\{\text { substitution }\} \\
& {[J \wedge(b \vee \neg p \vee q \vee r) \Rightarrow} \\
& (\neg b \vee(w l p . s .(J \wedge(b \vee \neg p \vee q \vee r)) \wedge w t o . s . p . q .(w e v . D O . q . r))) \wedge(b \vee \neg p \vee q \vee r)] \\
& \Leftarrow \quad\{[\neg b \wedge(\neg p \vee q \vee r) \Rightarrow(\neg b \vee \text { anything }) \wedge(b \vee \neg p \vee q \vee r)]\} \\
& {[J \wedge b \Rightarrow} \\
& (\neg b \vee(w l p . s .(J \wedge(b \vee \neg p \vee q \vee r)) \wedge w t o . s . p . q .(w e v . D O . q . r))) \wedge(b \vee \neg p \vee q \vee r)] \\
& \equiv \quad\{(47) \text {, calculus }\} \\
& \text { true }
\end{aligned}
$$

(End of proof)

Again, we give an example to demonstrate the use of the invariance theorem. The example is the loop

$$
D O=\text { do } i \neq 0 \rightarrow i:=i-1 \text { od }
$$

and we show

$$
[m \geq n \geq 0 \vee i<m \Rightarrow \text { wto.DO. }(i=m) .(i=n) \text {.false }] \quad .
$$

The choice for $J$ is $m \geq n \geq 0 \vee i<m$. Observe $[J \equiv J \wedge(b \vee \neg p \vee q \vee r)]$. The result follows from the invariance theorem for wto provided 


$$
[J \wedge b \Rightarrow \text { wto.s.p.q.(wev.DO.q.r)^wlp.s.J] } \quad .
$$

We deal with the last term first.

$$
\begin{array}{ll} 
& \text { wlp.s.J } \\
\equiv & m \geq n \geq 0 \vee i-1<m \\
\Leftarrow \quad & (m \geq n \geq 0 \vee i<m) \wedge i \neq 0
\end{array}
$$

In order to tackle the first term, we proceed with showing $[i \geq n \geq 0 \Rightarrow$ wev.DO. $(i=n)$.false $]$. We do so by applying the invariance theorem for wev with the following choices:

$$
\begin{aligned}
& I: \quad i \geq n \geq 0 \\
& t: \quad i \\
& C: \text { natural numbers }
\end{aligned}
$$

Observe $[I \wedge(i \neq 0 \vee i=n) \equiv I]$. The invariance theorem applies since

$$
\begin{aligned}
& {[I \wedge b \Rightarrow t \in C]} \\
& {[I \wedge b \wedge t=x \Rightarrow \text { wev. }(i:=i-1) .(i=n) .(I \wedge t<x)]}
\end{aligned}
$$

for all $x$. Both proofs are just a matter of substitution, and are omitted. This leaves us to show

$$
[J \wedge b \Rightarrow \text { wto.s.p.q. }(i \geq n \geq 0)] \quad .
$$

We have

$$
\begin{array}{ll} 
& \text { wto. }(i:=i-1) .(i=m) .(i=n) .(i \geq n \geq 0) \\
& (i \neq m \vee i=n \vee i-1=n \vee i-1 \geq n \geq 0) \wedge \\
& (i-1 \neq m \vee i-1=n \vee i-1 \geq n \geq 0) \\
\Leftarrow \quad & \\
\Leftarrow \quad & \\
& (m \geq m \geq n \geq 0 \vee i<m \\
& (m \geq 0 \vee i<m) \wedge i \neq 0
\end{array}
$$

which completes the proof.

\section{Concluding Remarks}

In this paper we have introduced new predicate transformers to express progress properties of programs. These predicate transformers were shown to be extensions of the familiar predicate transformers $w p$ and $w l p$. The inherent complexity of these new predicate transformers led to a large number of requirements or healthiness conditions to be imposed in order to pin down the characteristics of the predicate transformers. Verification of these healthiness conditions is a laborious task. It is difficult only for the case of the iterative command. As an alternative one might give an operational semantics of the program notation. In such an operational semantics, verification of the healthiness conditions would be easier, since induction over the syntax can be avoided. It also allows for a more rigorous (but non-trivial) proof of which solution of a defining equation must be chosen as the predicate transformer of the iterative command. Yet another advantage is that the 
definitions of the predicate transformers for the various commands that we have given in this paper can be derived from the operational semantics. In this paper we have chosen to follow the path that avoids the operational semantics since it makes the analysis cleaner: only the aspects that are relevant to our present purpose are involved. It also allows a more relaxed implementation of the programming language.

The application of our predicate transformers in the specification of of programs is mainly in the area of parallel programs. Although parallel composition is not part of our program notation, we can "simulate" parallelism by constructing a repetition of an alternative statement. Such a program is called an action system and was first introduced by Back and Kurki-Suonio in [1]. If the alternatives are further restricted to assignment statements and if a fair choice between the alternatives is postulated we end up with UNITY (cf. [2]).

Some recent papers have been published that deal with various predicate transformers. In [6] the predicate transformer "weakest leads to" is considered. The weakest leads to of a program and a predicate $q$ is the weakest predicate $p$ such that $p$ leads to $q$ holds for that program. No obvious relationship to the program's precondition exists. The relation to commands in a program notation is investigated for UNITY programs only. Because UNITY's individual statements are deterministic and because of the absence of sequential composition, the equations that result are slightly simpler than ours. In [8], Morris discusses a predicate transformer that is very similar to our wev/wlev, the main difference being that the intermediate states considered by Morris are only those states that directly precede the call of a recursive procedure.

The development of our predicate transformers seems to follow a pattern, and one might wonder whether it is possible to make the pattern explicit and specialize the pattern to the cases that we have discussed. We have not succeeded in doing so, the main problem being the fact that wev is the strongest solution of its defining equation (22) whereas wto is the weakest solution of its defining equation (43). This seems to be a formidable obstacle in coming up with a single pattern. One is referred to the proof of ( $\mathrm{T}^{\prime}$ ') for the iterative command (see last appendix) to see the complexity of linking a weakest solution to a strongest solution.

\section{Acknowledgement}

We are very grateful to one of the referees for insightful comments that helped us improve the presentation and that clarified some technical problems.

\section{Appendix - proofs for (E0) through (E5)}

In this appendix we give the proofs that the predicate transformers wlev and wev of the statements satisfy the requirements that we have imposed. Since most proofs are quite simple, we only consider the case of the repetition. Notice that (E1'), (E4') and (E5) follow from the other requirements. The proofs of (E0') and (E3') have been omitted because they are similar to the proofs for (E0) and (E3) respectively. 
$(\mathrm{E} 0):$

$$
\begin{array}{ll} 
& \begin{array}{c}
\text { wlev.DO.false.r } \\
\equiv \quad \\
\text { the weakest solution of } y:[y \equiv(b \vee r) \wedge \text { wlev.IF.false. } y]
\end{array} \\
\equiv \quad & \{(\mathrm{E} 0) \text { for IF }\} \\
& \text { the weakest solution of } y:[y \equiv(b \vee r) \wedge w l p . I F . y] \\
\equiv \quad & \{\text { definition of wlp.DO }\} \\
& \text { wlp.DO.r }
\end{array}
$$

(E1): $\quad z \wedge$ wlev.IF.q.y is a universally conjunctive function of $(z, y)$ i.e.

$$
\equiv \begin{aligned}
& \langle\forall z, y: z \in Z \wedge y \in Y: z \wedge \text { wlev.IF.q.y }\rangle \\
& \langle\forall z: z \in Z: z\rangle \wedge \text { wlev.IF.q. }\langle\forall y: y \in Y: y\rangle
\end{aligned}
$$

It follows that $h . z$, the weakest solution of (23), is a universally conjunctive function of $z$.

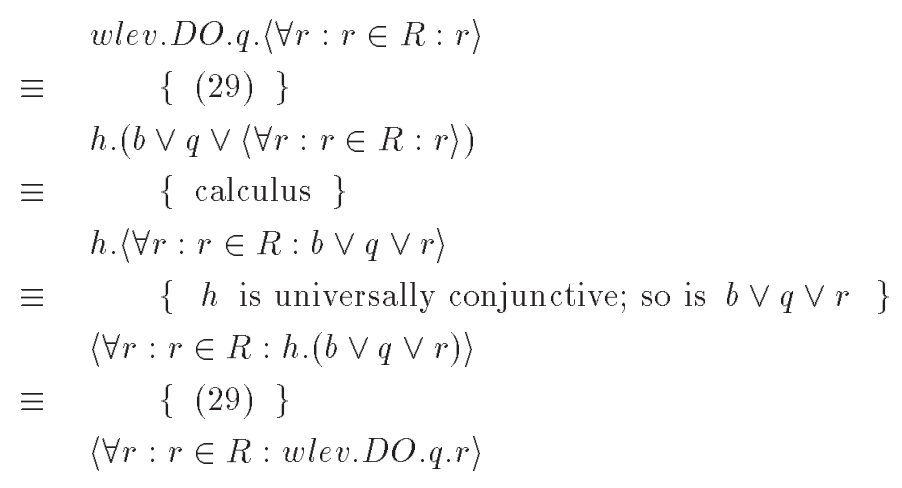

(E2):

$$
\begin{array}{ccc} 
& \text { wev.DO.q.r } \\
\equiv \quad & \{(30) ;(8)\} \\
& g .(\neg b \vee \text { wev.s.q.true }) \wedge h .(b \vee q \vee r) \\
\equiv \quad & \{[\text { true } \equiv b \vee q \vee \text { true }]\} \\
& \text { g. }((\neg b \vee \text { wev.s.q.true }) \wedge(b \vee q \vee \text { true })) \wedge h .(b \vee q \vee r) \\
\equiv \quad & \{(31) \text { and }(29)\} \\
& \text { wev.DO.q.true } \wedge \text { wlev.DO.q.r }
\end{array}
$$

(E3): In order to show that, given $\left[q \Rightarrow q^{\prime}\right]$, wlev.DO.q.r implies wlev.DO.q. $q^{\prime} \cdot r$ we use the fact that the latter is the weakest solution of its defining equation. According to Knaster-Tarski it is also the weakest solution of the equation in which equivalence is replaced by implication. We are left to show that wlev.DO.q.r is also a solution of the latter equation. 


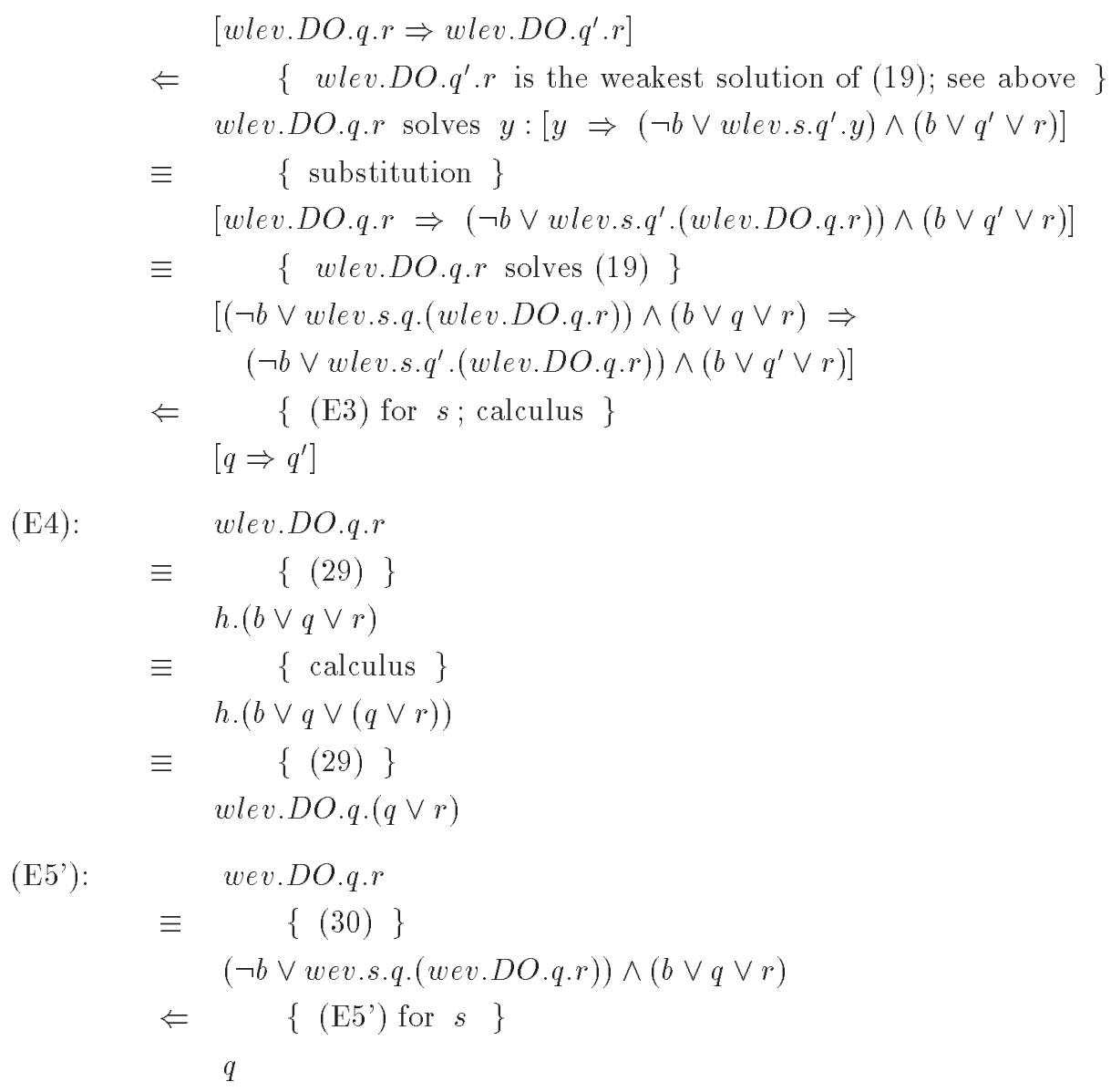

We draw attention to the fact that we can use the definitions of wlev.DO and wev.DO in terms of the function $h$ as long as the second argument, viz. $q$, is fixed. Since $q$ is hidden in the definition of $h$, we have to go back to the original equation when we analyze the dependence on $q$. For example, the proof of (E4) is valid since $h$ does not depend on $r$. The similar-looking argument

$$
\begin{array}{ccc} 
& \text { wlev.DO.q.r } \\
\equiv & \{(29)\} \\
& h .(b \vee q \vee r) \\
\equiv & \{\text { calculus }\} \\
& h .(b \vee(q \vee r) \vee r) \\
\equiv & \{(29)\} \\
& \text { wlev.DO. }(q \vee r) . r
\end{array}
$$

is invalid (and fortunately so) because $h$ does depend on $q$ and we, therefore, have a different function $h$ when the second argument of wlev is $q \vee r$.

\section{Appendix - proofs for (T0) through (T8)}

In this appendix we give the proofs that the predicate transformers wlto and wto of the statements satisfy the requirements that we have imposed. Again, we restrict attention to the loop. Notice that (T1'), (T4') and (T5) follow from the other requirements. The proofs of (T0'), (T3'), (T6'), (T7'), 
and (T8') have been omitted because they are similar to the proofs for the corresponding 'liberal' requirements. Remember that the $g$ and $h$ of (44) are the same as the $g$ and $h$ of (13).

(T0):

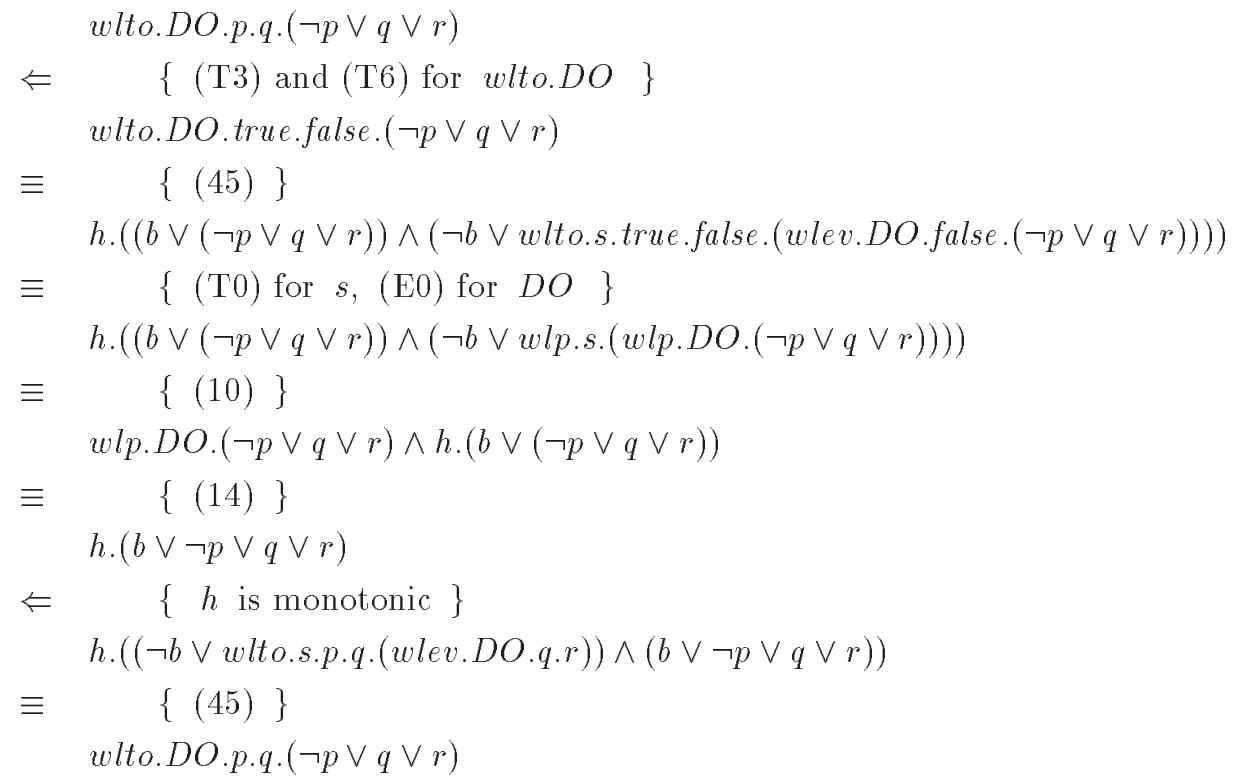

Observe that, in the first step of this proof, we have used (T3) and (T6). In the proofs thereof, we avoid references to ( $\mathrm{T} 0)$.

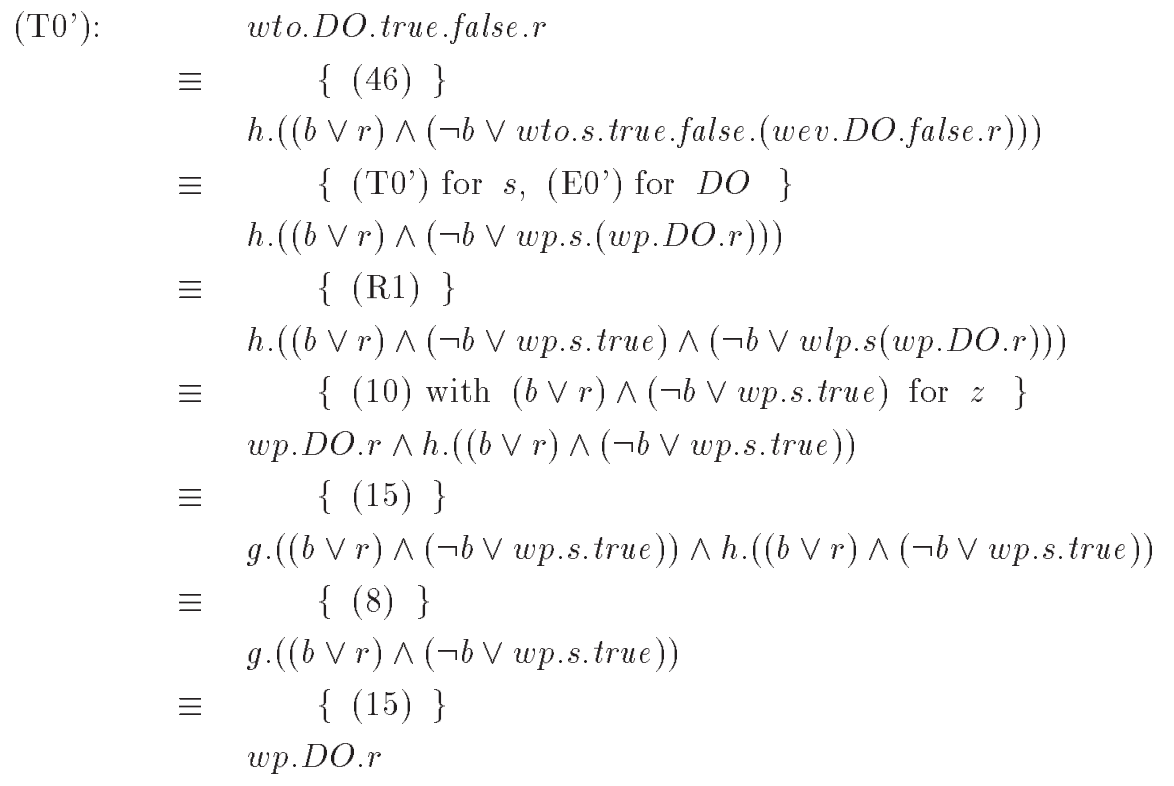


(T1):

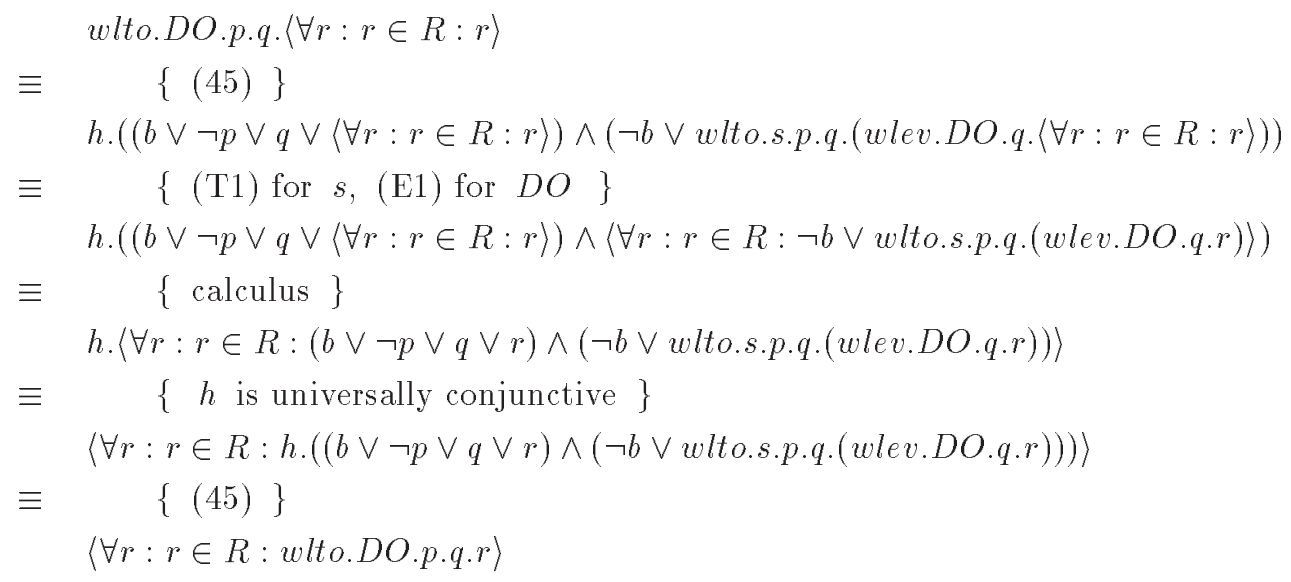

(T2):

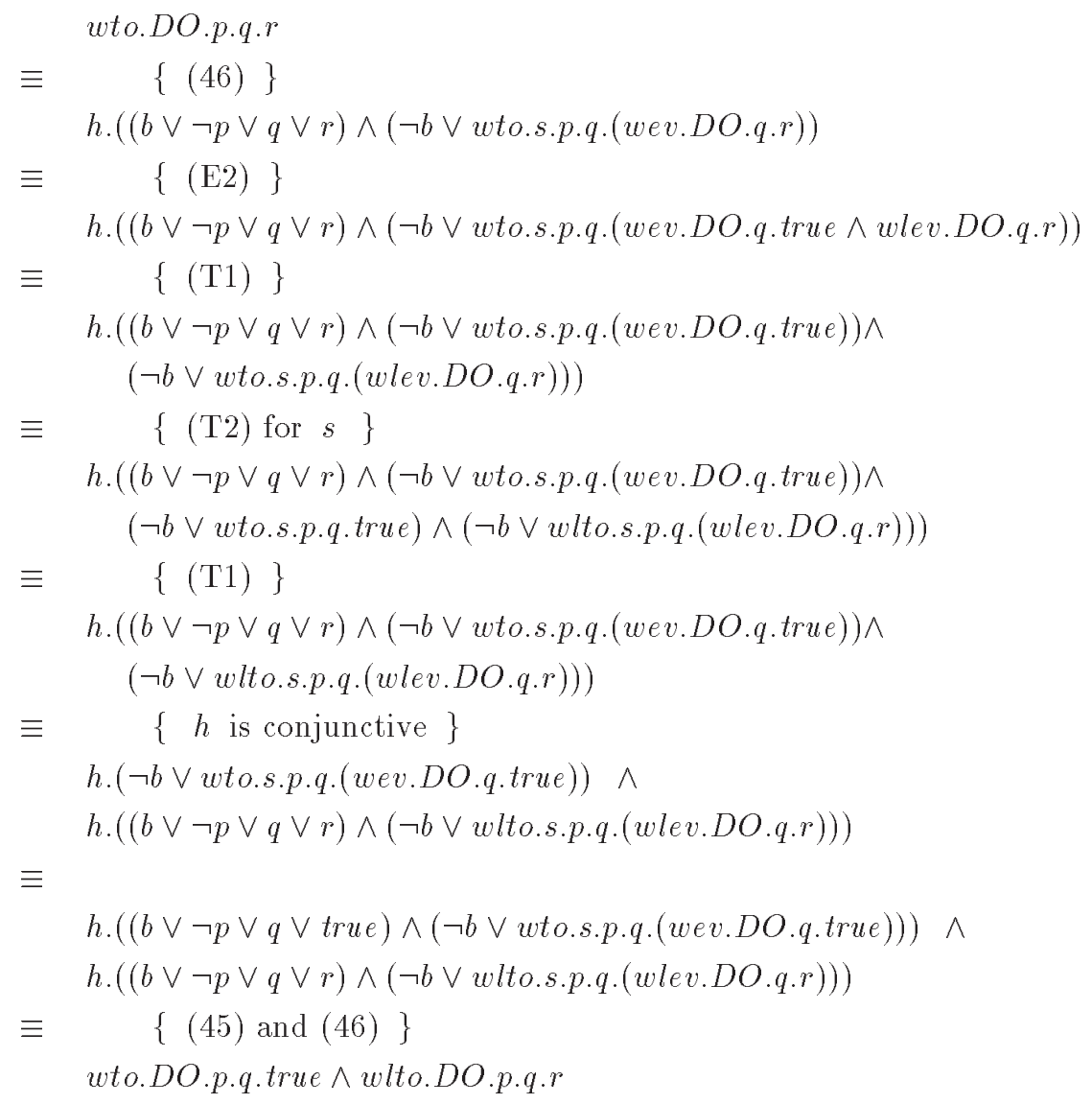


(T3):

$$
\begin{aligned}
& \text { [wlto.DO.p.q.r } \Rightarrow \text { wlto.DO.p.q. } . r] \\
& \equiv \quad\{(45)\} \\
& {[h .((b \vee \neg p \vee q \vee r) \wedge(\neg b \vee \text { wlto.s.p.q.(wlev.DO.q.r })) \Rightarrow} \\
& h .\left(\left(b \vee \neg p \vee q^{\prime} \vee r\right) \wedge\left(\neg b \vee \text { wlto.s.p. } q^{\prime} .\left(\text { wlev.DO. } q^{\prime} . r\right)\right)\right] \\
& \Leftarrow \quad\{h \text { is monotonic }\} \\
& \text { [wlto.s.p.q.(wlev.DO.q.r } \left.) \Rightarrow \text { wlto.s.p.q. } q^{\prime} \cdot\left(\text { wlev.DO. } q^{\prime} \cdot r\right)\right] \wedge\left[q \Rightarrow q^{\prime}\right] \\
& \Leftarrow \quad\{\text { (E3) and (T1) for DO }\} \\
& \text { [wlto.s.p.q.(wlev.DO.q.r }) \Rightarrow \text { wlto.s.p.q'.(wlev.DO.q.r })] \wedge\left[q \Rightarrow q^{\prime}\right] \\
& \Leftarrow \quad\{\text { (T3) for } s\} \\
& {\left[q \Rightarrow q^{\prime}\right]} \\
& \text { (T4): } \quad \text { wlto.DO.p.q.r } \\
& \equiv \quad\{(45)\} \\
& h .((b \vee \neg p \vee q \vee r) \wedge(\neg b \vee \text { wlto.s.p.q.(wlev.DO.q.r })) \\
& \equiv \quad\{(\mathrm{E} 4)\} \\
& \text { h. }((b \vee \neg p \vee q \vee(q \vee r)) \wedge(\neg b \vee \text { wlto.s.p.q.(wlev.DO.q. }(q \vee r))) \\
& \equiv \quad\{(45)\} \\
& \text { wlto.DO.p.q. }(q \vee r) \\
& \equiv \quad\{(46)\} \\
& \text { h. }((b \vee \neg p \vee p \vee r) \wedge(\neg b \vee \text { wto.s.p.p. }(\text { wev.DO.q.r })) \\
& \equiv \quad\left\{\left(\mathrm{T} 5^{\prime}\right) \text { for } s\right\} \\
& \text { h.true } \\
& \equiv \underset{\text { true }}{\{h \text { is universally conjunctive }\}} \\
& \text { (T6): } \quad\langle\forall p: p \in P: \text { wlto.DO.p.q.r }\rangle \\
& \equiv \quad\{(45)\} \\
& \langle\forall p: p \in P: h .((b \vee \neg p \vee q \vee r) \wedge(\neg b \vee \text { wlto.s.p.q.(wlev.DO.q.r })))\rangle \\
& \equiv \quad\{h \text { is universally conjunctive }\} \\
& \text { h. }\langle\forall p: p \in P:(b \vee \neg p \vee q \vee r) \wedge(\neg b \vee \text { wlto.s.p.q.(wlev.DO.q.r }))\rangle \\
& \equiv \quad\{(\mathrm{T} 6) \text { for } s\} \\
& \text { h. }((b \vee \neg\langle\exists p: p \in P: p\rangle \vee q \vee r) \wedge(\neg b \vee \text { wlto.s. }\langle\exists p: p \in P: p\rangle . q .(\text { wlev.DO.q.r })))) \\
& \equiv \quad\{(45)\} \\
& \text { wlto.DO. }\langle\exists p: p \in P: p\rangle . q . r
\end{aligned}
$$$$
\text { (T5'): } \quad \text { wto.DO.p.p.r }
$$

(T7): wlev.DO.q.r is the weakest solution of

$$
y:[y \Rightarrow(\neg b \vee \text { wlev.s.q.y }) \wedge(b \vee q \vee r)]
$$

and hence it suffices to show that a solution $w$ exists that is implied by the premise of (T7), i.e.

$$
\begin{aligned}
& {[w \text { lto.DO.p.q.r } \wedge \text { wlev.DO.p.r } \Rightarrow w]} \\
& {[w \Rightarrow(\neg b \vee \text { lev.s.q.w }) \wedge(b \vee q \vee r)]}
\end{aligned}
$$

By choosing

$$
[w \equiv \text { wlto.DO.p.q.r } \wedge \text { wlev.DO. }(p \vee q) . r]
$$

the first of these proof obligations follows from (E3). The second proof obligation is settled by 


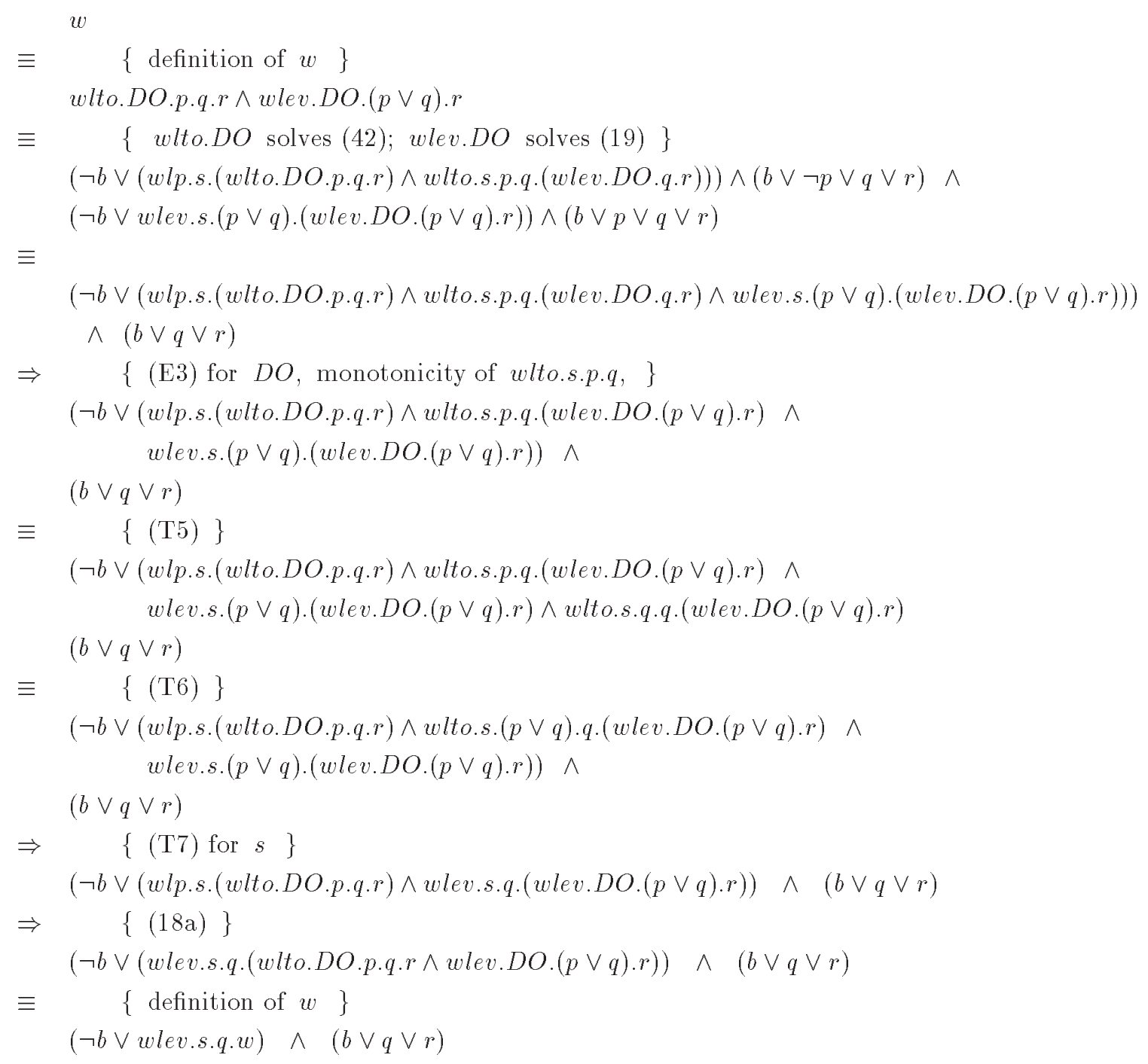

$\left(\mathrm{T} 7^{\prime}\right)$ : The proof of $\left(\mathrm{T} 7^{\prime}\right)$ is quite different from the proof of $(\mathrm{T} 7)$ and is, therefore, included. we have to show

$$
\text { [wto.DO.p.q.r } \wedge \text { wev.DO.p.r } \Rightarrow \text { wev.DO.q.r] }
$$

which is equivalent to

$$
[\text { wev.DO.p.r } \Rightarrow \neg \text { to.DO.p.q.r } \vee \text { wev.DO.q.r] } \quad \text {. }
$$

Since wev.DO.p.r is the strongest solution of its defining equation, it suffices to show that 
is also a solution of that same equation (with equivalence replaced by implication). Here is the proof.

$$
\begin{aligned}
& {[(\neg b \vee \text { wev.s.p. }(\neg \text { wto.DO.p.q. } r \vee \text { wev.DO.q.r })) \wedge(b \vee p \vee r)} \\
& \Rightarrow \neg w \text { to.DO.p.q.r } \vee \text { wev.DO.q.r] } \\
& \equiv \quad\{\text { calculus }\} \\
& {[(\neg b \vee \text { wev.s.p. }(\neg \text { wto.DO.p.q. } r \vee \text { wev.DO.q.r })) \wedge(b \vee p \vee r) \wedge \text { wto.DO.p.q.r }} \\
& \Rightarrow \text { wev.DO.q.r] } \\
& \equiv \quad\{\text { wto.DO.p.q.r solves (43) }\} \\
& {[(\neg b \vee \text { wev.s.p. }(\neg \text { wto.DO.p.q.r } \vee \text { wev.DO.q.r })) \wedge} \\
& (\neg b \vee(\text { wlp.s. }(\text { wto.DO.p.q.r }) \wedge \text { wto.s.p.q. }(\text { wev.DO.q.r }))) \wedge \\
& (b \vee p \vee r) \wedge(b \vee \neg p \vee q \vee r) \\
& \Rightarrow \text { wev.DO.q.r] } \\
& \equiv \quad\{(18 \mathrm{c})\} \\
& {[(\neg b \vee(\text { wev.s.p. }(\text { wev.DO.q.r }) \wedge \text { wlp.s. }(\text { wto.DO.p.q.r }) \wedge \text { wto.s.p.q.(wev.DO.q.r }))) \wedge} \\
& (b \vee(p \wedge q) \vee r) \\
& \Rightarrow \text { wev.DO.q.r] } \\
& \Leftarrow \quad\left\{\left(\mathrm{T} 7^{\prime}\right) \text { for } s \text {; calculus }\right\} \\
& {[(\neg b \vee \text { wev.s.q. }(\text { wev.DO.q.r })) \wedge(b \vee q \vee r) \Rightarrow \text { wev.DO.q.r }]} \\
& \equiv \quad\{\text { wev.DO.q.r solves }(20)\} \\
& \text { true }
\end{aligned}
$$

(T8): We have

$$
\begin{aligned}
& \text { wlto.DO.p.q.r } \wedge \text { wlto.DO.q.w.r } \\
& \equiv \quad\{\text { wlto.DO solves (42) }\} \\
& (\neg b \vee(\text { wlp.s. }(\text { wlto.DO.p.q.r }) \wedge \text { wlto.s.p.q. }(\text { wlev.DO.q.r }))) \wedge \\
& (\neg b \vee(\text { wlp.s. }(\text { wlto.DO.q.w.r }) \wedge \text { wlto.s.q.w.(wlev.DO.w.r }))) \wedge \\
& (b \vee \neg p \vee q \vee r) \wedge(b \vee \neg q \vee w \vee r) \\
& \Rightarrow \quad\{\text { calculus }\} \\
& (\neg b \vee(\text { wlp.s. }(\text { wlto.DO.p.q.r }) \wedge \text { wlp.s. }(\text { wlto.DO.q.w.r }) \wedge \\
& \text { wlto.s.p.q.(wlev.DO.q.r }) \wedge \text { wlto.s.q.w.(wlev.DO.w.r }))) \wedge \\
& (b \vee \neg p \vee w \vee r) \\
& \Rightarrow \quad\{(40 \mathrm{a}) ;(\mathrm{T} 7) \text { for } D O\} \\
& (\neg b \vee(\text { wlp.s.(wlto.DO.p.q.r }) \wedge \text { wlp.s. }(\text { wlto.DO.q.w.r }) \wedge \\
& \text { wlto.s.p.q.(wlev.DO.w.r }) \wedge \text { wlto.s.q.w.(wlev.DO.w.r }))) \wedge \\
& (b \vee \neg p \vee w \vee r) \\
& \Rightarrow \quad\{\text { conjunctivity of wlp.s; (T8) for } s\} \\
& (\neg b \vee(\text { wlp.s. }(\text { wlto.DO.p.q.r } \wedge \text { wlto.DO.q.w.r }) \wedge \text { wlto.s.p.w. }(\text { wlev.DO.w.r }))) \wedge \\
& (b \vee \neg p \vee w \vee r)
\end{aligned}
$$

which shows that wlto.DO.p.q.r $\wedge$ wlto.DO.q.w.r is a solution of (42), with equivalence replaced by implication. Since wlto.DO.p.w.r is the weakest solution of (42), the result follows.

\section{References}

[0] R.J.R. Back; On the correctness of refinement in program development; PhD-thesis, University of Helsinki, 1978. 
[1] R.J.R. Back, R. Kurki-Suonio; Decentralization of process nets with centralized control, 2nd ACM SIGACT-SIGOPS Symposium on principles of Distributed Computing, Montreal, Canada, August 1983, 131-142.

[2] K. Mani Chandy, J. Misra; Parallel Program Design: A Foundation, Addison Wesley, 1988.

[3] Edsger W. Dijkstra; A Discipline of Programming, Prentice-Hall, 1976.

[4] Edsger W. Dijkstra, Carel S. Scholten; Predicate Calculus and Program Semantics, SpringerVerlag, 1990.

[5] David Gries; The Science of Programming, Springer-Verlag, 1981.

[6] C.S. Jutla, E. Knapp, J.R. Rao; A Predicate Transformer Approach to Semantics of Parallel Programs; Proceedings of the 8th Annual ACM Symposium on Principles of Distributed Computing 1989, 249-263.

[7] Johan J. Lukkien; Parallel Program Design and Generalized Weakest Preconditions. Ph-D Thesis Groningen University, 1991.

[8] Joseph M. Morris; Temporal Predicate Transformers and Fair Termination; Acta Informatica, vol 27 (1990) $287-313$

[9] S. Owicki, L. Lamport; Proving Liveness Properties of Concurrent Programs; ACM TOPLAS, vol. 4, no. 3, July 1982, 455-495.

[10] A. Tarski; A lattice-theoretical fixpoint theorem and its applications; Pacific Journal of Mathematics, vol.5(1955), 285-309. 